\title{
Unsteadiness characteristics and three-dimensional leading shock structure of a Mach 2.0 shock train
}

\author{
Robin L. Hunt*, James F. Driscoll ${ }^{\dagger}$, and Mirko Gamba ${ }^{\ddagger}$ \\ University of Michigan, Ann Arbor, MI 48109
}

The structure and unsteadiness characteristics of a shock train in a Mach 2.0 ducted flow are studied. The shock train is generated and stabilized by a back-valve that imposes a desired back pressure. Despite nominally constant boundary conditions (i.e., inflow and outflow), the shock train fluctuates about its time-average position. High-speed schlieren imaging is used to quantify the amount of unsteadiness. The results show that the shock fluctuation speed and the magnitude of shock displacement are independent of back pressure. However, the angles of the leading shock lambda foot and the length of the leading shock Mach stem change as back pressure is increased indicating the shock structure transitions from oblique to normal as the shock train moves upstream. The structural changes of the shock train are reflected in time-averaged pressure profiles. As the shock train moves upstream the length of the shock train decreases and the pressure rise across the shock train increases. Finally, stereo particle image velocimetry is used to study the structure of the leading shock in a normal shock train (i.e., when the back pressure is high and pushes the shocks upstream). The velocity fields show a large separation region on the side-wall of the duct and a degree of axisymmetry that indicates a nominally conical shape. The overall structure of the leading shock is modeled as two truncated cones with the small ends coinciding to form the Mach stem.

\section{Nomenclature}

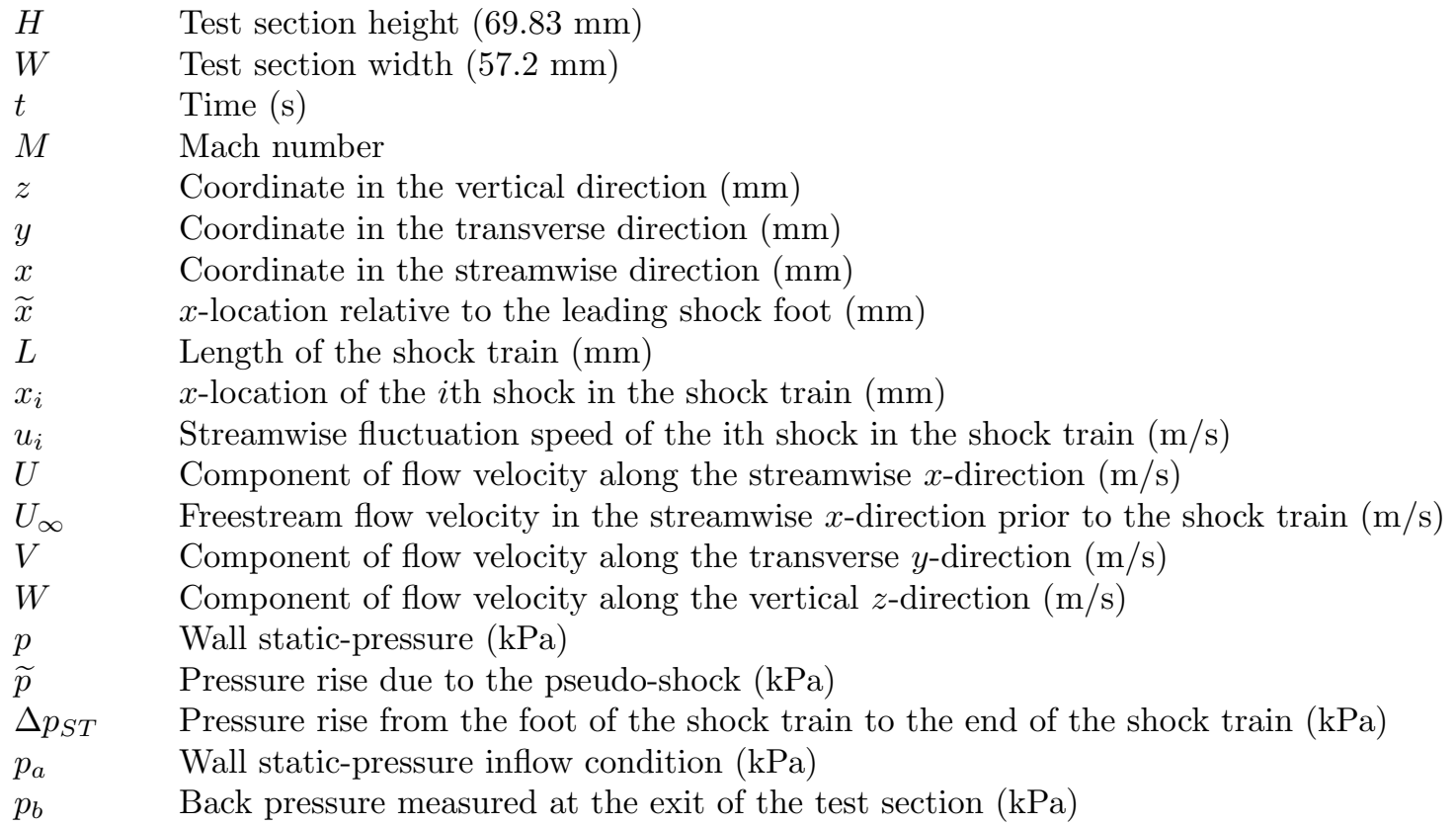

${ }^{*}$ Graduate Research Assistant, Dept. of Aerospace Engineering, AIAA member.

${ }^{\dagger}$ Arthur B. Modine Professor, Dept. of Aerospace Engineering, AIAA member.

$¥$ Assistant Professor, Dept. of Aerospace Engineering, AIAA member. 
$\alpha_{L} \quad$ Angle of the leading leg in the first shock lambda foot (deg)

$\alpha_{R} \quad$ Angle of the trailing leg of the first shock lambda foot (deg)

$s \quad$ Mach stem height $(\mathrm{mm})$

$\langle X\rangle \quad$ Time-average value of property $X$ (units of $X$ )

$X^{\prime} \quad$ Fluctuation component of property $X ; X^{\prime}=X-\langle X\rangle$ (units of $X$ )

$\sigma(X) \quad$ Standard deviation of property $X$ (units of $X$ )

$\max (X) \quad$ Maximum value of signal $X$

$P S D(X)$ Power spectral density of $X$ (units of $X^{2} / \mathrm{Hz}$ )

\section{Introduction}

In high-speed air-breathing engine designs, the isolator is a critical structure that separates the inlet from the combustor. An increased back pressure from the combustion process causes the formation of multiple coupled shock waves, and thus multiple coupled shock boundary layer interactions, in the engine isolator. This system of shocks is known as a shock train. The shock train slows the flow and provides the static pressure rise prior to the combustor, thus supplying suitable airflow for efficient combustion. Downstream of the shock train is the mixing region, where no shocks exist but the highly non-uniform velocity profile causes mixing that leads to additional pressure rise. Matsuo et al. ${ }^{1}$ termed the entire region from the beginning of the shock train to the end of the mixing region the pseudo-shock. Figure 1 shows two schematics, inspired by Matsuo et al., ${ }^{1}$ of different types of shock trains typically encountered in the pseudo-shock system: the normal and oblique shock trains. A normal shock train generally has a leading bifurcated normal shock followed by several non-bifurcated shocks. After each normal shock is a re-acceleration core flow region where the flow speed increases back to supersonic conditions. In an oblique shock train, right-running and left-running oblique shock waves are generated from opposite walls of the duct and cross to form an "X" pattern. The most upstream left- and right-running shocks are marked "L" and "R", respectively in figure 1(b). Multiple "X" structures form the shock train.

The dynamics of the shock train are complex and not fully understood. Even with nominally constant inflow and outflow isolator conditions, the fluid system exhibits inherent unsteadiness where the shock train fluctuates about its time average position. Studies have shown that increasing the Mach number tends to increase the shock fluctuation amplitude, with shock position displacements reaching up to a duct height for large Mach numbers. ${ }^{2-4}$ The existence of the shock train inherent unsteadiness leads us to define this mode of operation as quasi-steady state. The unsteady movement of the shock train is of practical importance because it may feed instabilities to the combustor and induce pressure fluctuations that generate noise and fluctuating wall loads, both of which need to be minimized..$^{5,6}$ Also, large fluctuation amplitudes can lead to premature engine unstart, where the shock system propagates upstream until it is disgorged from the inlet. When the shock train is ejected, a bow shock forms outside of the inlet leading to flow spillage and reduced mass flow rate through the engine. As a consequence, there is loss of engine thrust, significantly increased loads, and intense oscillatory flow. ${ }^{7-9}$ To date, much about the unsteady aspect of shock trains is not understood, including the fundamental physics that drive the unsteadiness. Better knowledge of the shock train dynamics would provide insight on engine control with the ultimate goal being the introduction of control algorithms to 1) reduce the system unsteadiness and thus increase the operating margin of the engine, 2) control structural properties such as the shock train length, and 3) better predict and prevent unstart.

To add to the complexity of the shock train, the physical structure of the system is highly threedimensional and is influenced by many parameters, including Mach number, boundary layer height, and duct geometry. ${ }^{1,6,10}$ While there are models to predict the general properties of the shock train like its length and pressure rise, the three-dimensionality of the shock train makes predicting the detailed structure very difficult. Previous experimental studies have suggested that the shock front is curved (i.e., the arrival of the corners precedes that of the centerline) and a large separation region is associated with the leading shock location. ${ }^{11,12}$ The accurate location of the leading shock is particularly important because it is often used in unstart detection algorithms and control methods. ${ }^{13-15}$ Handa et al. ${ }^{16}$ suggested that the curvature of the shock is the result interaction between two bifurcated shock waves developed on two perpendicularly adjacent walls. This configuration produces a thin boundary layer and higher Mach number flow in the corners of the duct following the leading shock. This raises the important question of where flow separation 
within the shock train might occur. As the amount of separation increases, the core flow becomes more restricted and the state of the boundary layer will affect the unstart dynamics. ${ }^{17}$ Side-wall effects have also proven important in computational models. Morgan et al. ${ }^{18,19}$ demonstrated the effects of inclusion or noninclusion of side-walls on the boundary layer growth and found that fully resolving the side-walls may result in unstart. While computational models are getting better at predicting shock train structure, there are still many aspects of shock trains that cannot be predicted with simulations. Thus, understanding the structure of the shock train, specifically the effects of side-walls and boundary layer separation, could aid 1) the design of a more robust and efficient engine, 2) the development of optimal methods for shock train leading edge detection in regards to unstart prediction and control algorithms, and 3) the validation computational studies.

The goal of this paper is to quantify the structural properties and unsteadiness characteristics of a Mach 2.0 shock train at different back pressures. In a realistic application, the back pressure varies depending on the operating conditions of the combustor and may change as the vehicle follows its desired flight trajectory. For example, changes in fueling scheme may be experienced. Thus, we are effectively determining the sensitivity of the shock train structure and unsteadiness to changes in the downstream flow condition. The specific objectives of the work are as follows:

1. Compare the position fluctuation magnitude and frequency content for the first four shocks in the shock train at a given back pressure;

2. Quantify the amount of shock position fluctuation and the shock speed as a function of back pressure;

3. Determine how structural properties of the shock train, including the Mach stem height and shock angles, change with back pressure;

4. Examine the time-average pressure profiles for different back pressures by quantifying the shock train length and the pressure rise across the shock train;

5. Utilize stereo particle image velocimetry to evaluate the amount of separation under the leading shock and to evaluate the leading shock structure.

\section{Experimental setup}

\section{A. Wind tunnel facility}

The current experiments are performed in a suction type wind tunnel at the University of Michigan. A schematic diagram of the wind tunnel is shown in figure 2. Air enters the wind tunnel intake and then passes through a flow conditioning section containing honeycomb meshes. A one-sided converging-diverging nozzle is used to produce a nominal freestream Mach number of 2.0 in the test section of the wind tunnel. The low-aspect ratio test section has a constant, rectangular cross-section measuring $57.2 \mathrm{~mm} \times 69.8 \mathrm{~mm}$ $(W \times H)$. A right-handed coordinate system is used for this work. The $x$ coordinate direction is oriented streamwise with $x=0$ at the throat of the nozzle. The $z$-direction is normal to the bottom-wall. The origin is located on the lower right corner of the duct cross-section as one looks downstream.

The effective inflow conditions of the Mach 2.0 supersonic flow are summarized in table 1 . The nominal Mach number was verified using Pitot pressure measurements. The freestream flow speed was measured for this paper using stereoscopic particle image velocimetry (see section III C). The stagnation pressure and temperature are determined prior to every run by measuring the room conditions. In addition, the inflow static pressure is measured during every run with a MKS 626C Baratron at location (a) in figure 2. The remaining parameters that are not directly measured are estimated using the isentropic flow relations for the given Mach number.

\section{B. Generating the shock train}

A shock train is produced by partially closing a butterfly valve separating the diffuser to the vacuum chamber. Here, we refer to this valve as the control valve of the wind tunnel. The reduced area for airflow increases the pressure in the diffuser and a shock structure is formed in the test section to match the pressure increase. Further closing the valve pushes the shock train more upstream. 


\begin{tabular}{lc}
\hline Nominal Mach number & 2.0 \\
Flow speed, m/s & 505 \\
Unit Reynolds number, $\mathrm{m}^{-1}$ & $1.4 \times 10^{7}$ \\
Stagnation pressure, $\mathrm{kPa}$ & 98.1 \\
Stagnation temperature, $\mathrm{K}$ & 294 \\
Static pressure, $\mathrm{kPa}$ & 11.91 \\
Static temperature, $\mathrm{K}$ & 160 \\
Density, $\mathrm{kg} / \mathrm{m}^{3}$ & 0.28 \\
Viscosity, N-s $/ \mathrm{m}^{2}$ & $1.11 \times 10^{-5}$ \\
\hline
\end{tabular}

Table 1. Summary of test section free stream inflow conditions.

Two capacitance manometers (MKS 626C Baratron) are used to measure the pressure at the beginning and end of the test section. The manometer accuracy is $0.25 \%$ of the reading and the response time is about $0.2 \mathrm{~ms}$. Location (a) in figure 2 is the position where the inflow conditions to the shock train are defined and measured. This manometer is downstream of the diverging portion of the nozzle and within the constant area cross-section. The second manometer measures the static pressure just upstream of the diffuser, $p_{b}$ (see location (b) in figure 2), and is referred to as the back pressure of the shock train. The back pressure values reported here are typically normalized by the inflow pressure, $p_{a}$. Further closing the control valve will increase the back pressure and thus, the pressure ratio, $p_{b} / p_{a}$. In this paper we consider shock trains with pressure ratios between 2.7 and 3.5. The back pressure cannot be raised too far above the upper bound presented in this paper because the shock train moves into the diverging section of the wind tunnel tunnels and tunnel unstart may occur.

\section{Pressure measurements}

The side-wall and bottom-wall plug are replaced with aluminum pieces with pressure taps for low-speed static pressure measurements. The pressure taps are located along the centerline, $y=W / 2$ and $z=H / 2$, for the bottom- and side-wall, respectively. Wall pressure taps are spaced $12.7 \mathrm{~mm}$ apart for high spatial-resolution results. The recess-mounted pressure tap diameter is $0.8 \mathrm{~mm}$ and the tap length is $7.6 \mathrm{~mm}$. Stainless-steel tubing (inner diameter of $0.8 \mathrm{~mm}$ and outer diameter of $1.6 \mathrm{~mm}$ ) is press-fitted into the walls and then Tygon B-44-4X flexible tubing (inner diameter of $1.6 \mathrm{~mm}$ and outer diameter of $3.2 \mathrm{~mm}$ ) is used to connect the pressure taps to a differential pressure sensor array scanner.

Measurements are made with a Scanivalve DSA3217 and three NetScanner model 9116 digital sensor arrays at a rate of $20 \mathrm{~Hz}$. The inflow static pressure on the top-wall (see location (a) in figure 2) is used as a common reference pressure for all four pressure scanners. Absolute pressure measurements were performed at this location with two different transducers: 1) a pressure gauge (Omega model DPG2001B-30A, with an accuracy of $0.5 \mathrm{kPa}$ ); or 2) a capacitance manometer (MKS Baratron $626 \mathrm{C}$, with an accuracy of $0.25 \%$ of the reading).

\section{Cinematographic schlieren imaging}

The wind tunnel side-walls are replaced with Borosilicate glass pieces for optical access along the entire length of the test section. A z-type schlieren setup with a horizontal knife-edge is used to capture vertical density gradients in the flow. The light source was fabricated in-house and uses a high brightness LED (Luminus SBR-70) for continuous illumination. Images are recorded with a Phantom v711 camera at a rate of $10 \mathrm{kHz}$ with an exposure time of $1 \mu \mathrm{s}$. The camera field of view covers the entire test section height and approximately 9.5 in $(241 \mathrm{~mm})$ in the streamwise direction. The image resolution is $3.4 \mathrm{px} / \mathrm{mm}$.

Figure 3 shows an example of an instantaneous schlieren image of the Mach 2.0 shock train with a back pressure ratio of 3.16. Flow is from left to right. The leading shock has a normal structure, demonstrated by the Mach stem in between two very large lambda feet. Morphological features visible in the raw schlieren images are quantified automatically using an edge detection algorithm. The first step of the edge detection algorithm is to apply a Sobel filter to find edges in the image. Next, a median filter is applied to remove 
most of the edges that appear due to freestream turbulence. Figure 4 is an example of the resulting binary image after the median filter. The angles and locations of the shocks are found by fitting lines to specified areas of white pixels. In addition, a Harris corner detector is used to find the corner points where the shock legs intersect (i.e., the triple point). In this paper we examine the following morphological features labeled in figures 3 and 4 :

- Mach stem height, $s$ : defined as the difference between upper and lower triple point $z$-locations on the leading shock;

- Leading shock location, $x_{1}$ : defined the $x$-location of the Mach stem on the leading shock;

- Shock angles, $\alpha_{L}$ and $\alpha_{R}$ : defined as the angle of the leading and trailing legs of the first shock lambda foot referenced from the horizontal direction;

- Downstream shock locations, $x_{2}, x_{3}$, and $x_{4}$ : defined as the intersection points of subsequent shocks with the top-wall boundary layer. The analysis conducted here has also been conducted for morphological points defined on different points along the shock train; however, the conclusions drawn did not change.

By identifying these features in each frame of the schlieren image sequence, we can obtain time histories of the location and structure of some of the shock train components. In section IIIA we analyze the time history traces of the shock position to quantify the shock train unsteadiness. In section IIIB we consider the shock angles and Mach stem height to examine the physical structure of the shock train and how it transitions from a normal to an oblique shock train.

\section{E. Stereo particle image velocimetry}

Stereoscopic particle image velocimetry measurements are collected to quantify all three components of the flow velocity on a plane perpendicular to the flow (i.e., a cross-sectional plane parallel to the $y-z$ plane). The data plane is located at $x=497 \mathrm{~mm}$, which is the nominal position of the leading shock in the shock train for a pressure ratio of 3.5. Measurements were made using the setup shown in figure 5. A portable Laskin nozzle aerosol generator (ATI Model TDA-4B) was used to seed the flow with polydispersed submicrometer particles composed of poly-alpha olefin oil with a density of $819 \mathrm{~kg} / \mathrm{m}$. The estimated mean particle diameter is $0.7 \mu \mathrm{m}$, which is found to be adequately small to track the flow. ${ }^{20}$ Two interline transfer charge-coupled device cameras (SensiCam PCO) recording at $3.33 \mathrm{~Hz}$ with a resolution of $1280 \times 1024$ pixels were used in forward-scattering stereoscopic configuration. The cameras were oriented at $33^{\circ}$ relative to the measurement plane. The cameras were equipped with a Sigma 70-300 $\mathrm{mm} \mathrm{f/4-5.6} \mathrm{apochromatic} \mathrm{macro} \mathrm{lens.} \mathrm{The} \mathrm{double-}$ pulse illumination of the flow was provided by a pair of low-repetition-rate frequency-doubled Nd:YAG lasers producing an output of a $532 \mathrm{~nm}$ beam with a total energy of $200 \mathrm{~mJ} /$ pulse. The lasers were triggered at 10 $\mathrm{Hz}$ with a time delay of $600 \mathrm{~ns}$ between the two pulses and with a pulse duration of about $10 \mathrm{~ns}$. The effective time delay between pulses was measured with a fast response photodiode (Thorlabs DT10A, 1 ns response time) and a digital oscilloscope (LeCroy Waverunner 6030, $350 \mathrm{MHz}$ ). Both laser beams were sent through a combination of cylindrical lenses to generate the illumination sheet (see figure 5). L1 is a cylindrical lens focusing in the horizontal plane, L2 is a cylindrical lens expanding the beam in the vertical plane and L3 is a focusing cylindrical lens collimating the beam in the vertical plane. In this configuration, the laser sheet is perpendicular to the flow and illuminates part of the wind tunnel cross-section. The beam width, measured using the scanning knife-edge method, was $1.25 \pm 0.25 \mathrm{~mm}$, which is approximately four times the particle displacement in the freestream $(\approx 300 \mu \mathrm{m})$ in $600 \mathrm{~ns}$.

The LaVision DaVis 8 software was used for the acquisition of the measurement and the processing of the data. The three-component velocity fields were reduced from the particle images using a multipass scheme. Two passes were first conducted with a $64 \times 64$ pixel window size with $75 \%$ overlap, followed by two passes at a reduced $32 \times 32$ pixel window size with Gaussian weighting and $75 \%$ overlap. The final $32 \times 32$ pixel window size corresponds to a projected physical size of about $1.6 \times 1.6 \mathrm{~mm}$. The entire vector field spans a physical region of $31 \times 31 \mathrm{~mm}$ with a vector spacing of $0.4 \times 0.4 \mathrm{~mm}$. Post processing within multiple passes included deleting a vector if its correlation value was less than 0.8 as well as removing groups with less than 5 vectors. In addition, vectors with a first to second correlation peak ratio less than 1.1 were removed. Valid vectors were found more than $95 \%$ of the time. Missing or rejected vectors were interpolated using the method by Garcia. ${ }^{21}$ 


\section{Results}

\section{A. Statistics on the shock position unsteadiness}

In this section we consider the time variation of the shock positions to investigate the inherent unsteadiness of the system. We use schlieren images to quantify the position of shock waves and determine how the unsteadiness statistics change with increased back pressure. An example time trace of the shock position fluctuations for a short $10 \mathrm{~ms}$ period is plotted in figure 6 . The back pressure ratio, $p_{b} / p_{a}$, is equal to 3.16 for the case plotted.

First, we will compare the motion of shocks 1-4 in the shock train system for the back pressure case of $p_{b} / p_{a}=3.16$. The probability density functions (PDF) of the shock position fluctuation amplitudes are plotted in figure 7(a). All of the PDFs are approximately symmetric. Also, it is evident that all four shocks have approximately the same maximum fluctuation amplitude $(\approx 17 \mathrm{~mm})$ and fluctuation standard deviation $(\approx 5 \mathrm{~mm})$. The shock speeds are calculated from the time-history measurement of the shock position using the central difference method of adjacent points. Figure 7(b) shows the probability density function of the shock speeds. Unlike the shock displacement distribution, the speed distributions for each shock are different. The narrow probability distribution of the leading shock speed indicates that this shock exhibits lower speeds more often. The downstream shocks progressively exhibit higher speeds, broadening the distribution. The shock speed has also been calculated using alternative schemes, such as a forward difference, Richardson extrapolation, and least squares schemes, but nearly identical results are obtained.

The power spectral densities of the shock position fluctuations are shown in figure 8 for the case of $p_{b} / p_{a}=3.16$. All of the spectra are relatively broadband and the majority of the power is confined to low frequencies. The spectrum of $x_{1}^{\prime}$ has no significant local modes and decays with a slope of $1 / f^{2}$. Shocks that are further downstream have more content at the higher frequencies, raising the tail of the spectrum. Downstream shocks also demonstrate preferred modes indicating specific frequencies are more prominent in the shock motion (see labels in figure). For instance, the spectrum of $x_{2}^{\prime}$ has a bulge in frequency content around $500 \mathrm{~Hz}, 2.0 \mathrm{kHz}$, and $3.0 \mathrm{kHz}$. The modes at $\approx 500 \mathrm{~Hz}$ and $\approx 2 \mathrm{kHz}$ persist through the third and fourth shock power spectra. The $3 \mathrm{kHz}$ peak is apparent in the third shock power spectrum but has diminished by the fourth shock. While all four shocks have approximately the same distribution of fluctuation amplitudes they exhibit different fluctuation frequency content.

The above analysis shows the unsteadiness statistics and frequency content for a single back pressure ratio, $p_{b} / p_{a}=3.16$. The locations of shocks 2,3 , and 4 were not tracked for several of the runs because they were located outside the field of view. However, similar results were obtained over the smaller range of back pressure ratios where the downstream shocks were visible.

Next, we will consider the variation in unsteadiness quantities at different back pressure ratios. As mentioned in section IIB, the back pressure depends on the position of the control valve and in turn, the shock train position (i.e., its length) is dependent on back pressure. In figure 9 the time-average shock locations are plotted for the different back pressure ratios considered, with each symbol representing the result from individual repetition of the experiment. All four time-average shock locations move upstream linearly with increased back pressure. For any given back pressure ratio, the spacing between consecutive shocks decreases along the length of the shock train (i.e., $x_{2 t}-x_{1}=74 \mathrm{~mm}>x_{3}-x_{2 t}=47 \mathrm{~mm}>x_{4}-x_{3}=44 \mathrm{~mm}$ ). In addition, the spacing of shocks appears to be independent of back pressure.

Statistics on the leading shock position fluctuations and shock speed are plotted against $p_{b} / p_{a}$ in figures 10 and 11, respectively. Maximum values are represented by the circular symbols and standard deviations by the triangular symbols. The results suggest that the leading shock position unsteadiness and shock speed are fairly independent of back pressure. The instantaneous deviation of the shock position from its time-average position can be as large as 0.4 tunnel heights for the Mach 2.0 shock train. The leading shock can also reach speeds up to $16 \mathrm{~m} / \mathrm{s}$. Similar results are obtained for shocks 2,3 , and 4 . For each shock, the position fluctuation and speed statistics are independent of back pressure.

\section{B. The normal-oblique transition process}

In this section we consider the time-average structural features of the shock train quantified using the schlieren images. Specifically, we will analyze how the leading shock angles and Mach stem height change with back pressure ratio. Finally, we will present the time-average pressure profiles for the different shock train cases. We will consider how the pressure profile changes with back pressure by evaluating the shock 
train length and pressure rise across the shock train.

Figure 12 shows the time-average angles of the leading and trailing legs of the first shock lambda foot, $\left\langle\alpha_{L}\right\rangle$ and $\left\langle\alpha_{R}\right\rangle$, versus the back pressure ratio for the Mach 2.0 shock train. Recall that the angles are defined from the horizontal $x$-direction as shown in figure 3 . The standard deviation of the shock angle for any given case is approximately $1.6^{\circ}$. There is a clear linear trend in both the leading and trailing shock leg angles despite some run-to-run variation. Increasing the back pressure (or equivalently moving the shock train upstream) causes the angle of the leading shock leg in the lambda foot to increase. Simultaneously, the angle of the trailing shock leg decreases. Therefore, as the shock train moves upstream, the leading shock in the lambda foot becomes stronger and the trailing shock becomes weaker.

Figure 13 shows a plot of the time-average Mach stem height, $\langle s\rangle$, as a function of back pressure. The average Mach stem height increases non-linearly with back pressure. Thus, at low back pressures a Mach stem is not present in most instances; thus the leading shock can be considered oblique. At the highest back pressure, the Mach stem averages $9 \mathrm{~mm}$ long so the system can be defined as a normal shock train (with a significantly large foot). Contrary to the mean result, the instantaneous Mach stem height does not correlate with the instantaneous shock position during the unsteady motion. This indicates that there may be variables other than back pressure that influence the shock train structure during the normal-to-oblique transition process.

The probability density function of the Mach stem height is shown in figure 14. As the back pressure is increased, the distribution transitions from being strongly peaked at zero (oblique shock train) to having a broad, flat shape at intermediate values, and then finally having a more bell-shaped distribution at high back pressure ratios (normal shock train). During the transition process at intermediate values of back pressure, the shock train cannot be clearly defined as normal or oblique because the Mach stem height has a large amount of fluctuation.

The observations of figures 13 and 14 show that the leading shock in the Mach 2.0 shock train exhibits an oblique-to-normal transition process as back pressure is increased. Based on the literature, the incoming flow Mach number is the most influential parameter that typically determines the shock train regime. ${ }^{1,10,22}$ The degree of flow confinement, defined as the ratio of boundary layer thickness to test section half height, is generally found to have a secondary effect on shock train structure. ${ }^{6,11,23}$ In the current study, changing the back pressure moves the shock train to a different position in the wind tunnel, thus effectively altering the boundary layer height and confinement ratio that the shock train experiences. Figure 15 is a regime diagram developed by collecting the results of several prior studies. The results show that normal shock trains (blue symbols) exist at low Mach numbers and oblique shock trains (green symbols) form at Mach numbers above 1.8-2.2. Within the transition regime (Mach 1.8 to 2.2) both oblique and normal shock waves have been observed. The upper and lower cases of confinement for the current study are shown as (red) asterisks.

Finally, we consider the time-average pressure distribution of the shock train. Figure 16(a) shows the time-average pressure profile along the test section for multiple back pressure ratios. The solid lines represent the side-wall measurements (taken along $z=H / 2$ ) and the dashed line represent bottom-wall measurements (taken along $y=W / 2$ ). The shape of the Mach 2.0 side-wall pressure profile changes as back pressure is increased. The lowest back pressure case (i.e., an oblique shock train) has a non-monotonic pressure profile with many distinct steps and regions of decreasing pressure. The regions of decreasing pressure could be due to the flow re-accelerating after each shock. The highest back pressure case (i.e., a normal shock) has a smooth, monotonically increasing pressure profile. The bottom-wall pressure profiles are roughly the same as their side-wall counterparts but are smoother. Note that the beginning of the bottom-wall pressure rise precedes the side-wall measurements by less than $0.6 \mathrm{H}$. This result is unexpected because the schlieren images show a substantial lambda foot that would induce a pressure rise on the bottom-wall much earlier than the Mach stem would induce a pressure rise on the side-wall. The closer (than expected) pressure profiles indicate that the leading shock might have a three-dimensional structure that cannot be visualized with the schlieren method. We will discuss this further by analyzing the particle image velocimetry measurements in section IIIC.

To better visualize the effects of changing back pressure, the pressure profiles of figure 16(a) are shifted so that beginning of the shock train pressure rise starts at the origin as shown in figure 16(b). In the early portion of the system, the slope of the pressure rise is highest and the profiles collapse to approximately the same curve. After $2 H$ in the shifted $x$-direction the pressure profiles begin to diverge such that the lower back pressure cases have a shallower slope.

In the literature, the shock train region is traditionally identified visually using schlieren or shadowgraph 
imaging. These methods can be limited by the field of view and the results can be subjective. By measuring the time-average pressure profiles we propose more objective methods for defining the end of the shock train and the beginning of the mixing region. We will discuss two methods of defining the shock train region using: (1) variation of pressure in the $x$-direction and (2) the variation of pressure in time. In the first method, the derivative of pressure with respect to $x$ (i.e., the slope of the pressure profile) is calculated using the central difference method of adjacent points. An example of the resulting profile for a single back pressure case $\left(p_{b} / p_{a}=2.91\right)$ is shown in figure $17(\mathrm{a})$. As discussed previously, the steps in the pressure profile are associated with the flow being slowed after each shock and subsequently reaccelerated prior to the next shock in the system. Any pressure profile steps will produce local peaks in the pressure derivative. Thus, the shock train region is indicated by peaks in the pressure derivative. In the mixing region, no shocks exist and the pressure changes gradually. Mathematically, we define the beginning and end of the shock train as the first and last points, respectively, where the slope of the time-average pressure profile is above a value of $0.05 \mathrm{kPa} / \mathrm{mm}$. The red and blue markers in figure $17(\mathrm{a})$ indicate the beginning and end of the shock train, respectively, found using the pressure derivative method.

Alternatively, the shock train region can be identified using the the variation of pressure in time. For example, figure $17(\mathrm{~b})$ is constructed for the case of $p_{b} / p_{a}=2.91$ by finding the standard deviation of each pressure transducer time trace. The inherent unsteadiness of the shock train system will lead to variation in the pressure measurements as shocks fluctuate over a transducer. Thus, the peaks in pressure standard deviation are due to the unsteady shock motion. The standard deviation is lower in the mixing region where shocks do not exist. For this method, the beginning of the shock train is defined as the first point with a standard deviation above $0.05 \mathrm{kPa}$. The end of the shock train is defined as the last point with a standard deviation above $0.35 \mathrm{kPa}$. The beginning and end locations are marked in figure $17(\mathrm{~b})$ by the red and blue symbols, respectively. Figures 17(a) and 17(b) were used as examples of the two methods discussed. Figure 17 illustrates where the beginning (red markers) and end (blue markers) of the shock train are on the original pressure profile for both methods. With this information we can determine the pressure rise across the shock train, $\Delta p_{S T}$, and the length of the shock train, $L$ (see labels in figure $17(\mathrm{c})$ ).

The above procedure for locating the beginning and end of the shock train is repeated for multiple cases with different back pressures. Figures $18(\mathrm{a})$ and $18(\mathrm{~b})$ plot the pressure rise across the shock train and the shock train length, respectively, versus the pressure ratio, $p_{b} / p_{a}$. The green and black markers are the results of the pressure standard deviation and pressure derivative methods, respectively. For both methods, as the shock train is pushed upstream (i.e., $p_{b} / p_{a}$ increases) the pressure rise across the shock train increases and the length of the shock train decreases in addition to the leading shock structure becoming more normal. Generally, the length of the shock train is between 3-4 $H$ and the pressure rise across the shock train is between 19-24 kPa. The constant area duct is not long enough to measure the pressure at the end of the pseudo-shock. However, we estimate this pressure as $80 \%$ of the pressure rise across a single normal shock according to the results of previous work in the literature. ${ }^{1}$ Thus, for the current experiments the pressure rise due to the pseudo-shock is approximately $48 \mathrm{kPa}$ and the pressure rise across the shock train accounts for $\sim 40-50 \%$ of the entire pressure rise.

\section{Particle image velocimetry of the leading shock}

The schlieren images have provided insight on the two-dimensional projected structure of the shock train. In this section we present stereo particle image velocimetry (SPIV) measurements of the leading shock in the shock train in order to: 1) quantify all three components of velocity; 2) evaluate the amount of separation under the leading shock lambda foot; and 3) develop a three-dimensional representation of the leading shock structure. The laser sheet is oriented perpendicular to the flow so that we obtain velocity fields of the duct cross-section (i.e., the viewer's perspective as they look upstream through the test section). A single cross-sectional measurement plane located at $x=497 \mathrm{~mm}$ for the case of $p_{b} / p_{a}=3.5$ is considered. This plane is approximately located slightly upstream of the first shock Mach stem. However, because the shock fluctuates around its mean location by up to $\pm 0.2 \mathrm{H}$, the different instantaneous velocity field measurements are representative of different locations along the leading shock. By changing to a fixed shock reference frame we can determine the location of individual measurement planes relative to a stationary shock train structure seen in a shclieren image. Changing the reference frame allows us to obtain an approximate representation of the flow field around the first shock of the system.

First, the empty tunnel (i.e., when no shock train is present) measurements are presented to give an initial characterization of the undisturbed flow field. Figures 19(a), 19(b), and 19(c) are time-average results 
of the streamwise $(U)$, transverse $(V)$, and vertical components $(W)$ of velocity, respectively, when the shock train is situated far downstream of the measurement plane. Thus, these are the flow velocities without any influence from the shock train. Each time-average velocity field is calculated by averaging 300 images taken over three different runs. The vertical dashed line indicates where the test section half width $(y=W / 2)$ is located. In addition, the upper limit of the vertical axis is equal to the test section half-height $(z=H / 2)$. Due to interference from laser sheet reflections, the regions closest to the side- and bottom-walls are omitted. As seen in figure 19(a), the streamwise velocity in the core flow is approximately $U_{\infty}=505 \mathrm{~m} / \mathrm{s}$. The magenta contour line indicates the local boundary layer thickness $\left(0.99 U_{\infty}\right)$. Note that the boundary layer on both the side- and bottom-walls quickly thickens in the corner region. The corner also impacts the transverse velocity in figure $19(\mathrm{~b})$, as demonstrated by the low-speed $(\approx 10 \mathrm{~m} / \mathrm{s})$ fluid movement directed away from the corner. In figure $19(\mathrm{c})$, there is a slight upwards velocity $(\approx 10 \mathrm{~m} / \mathrm{s})$ evident in the vertical component of the flow velocity. From time-average schlieren images, we know that this small upwards velocity is due to the presence of a weak, uncanceled wave originating from the one-sided converging-diverging nozzle.

For the next part of the study, the control valve is partially closed to set the back pressure ratio, $p_{b} / p_{a}$, to approximately 3.5. At this back pressure the shock train is positioned in the test section such that the time-average location of the leading shock Mach stem is just downstream of the SPIV measurement plane (i.e., $x_{1} \approx 505 \mathrm{~mm}$ ). At this location, the leading shock has an average Mach stem height of approximately 7 $\mathrm{mm}$. Due to the inherent unsteadiness of the shock train system, the leading shock fluctuates about the SPIV measurement plane, allowing us to effectively measure different locations under the leading shock lambda foot. If we change to a reference frame with a fixed shock train location, then the measurement plane moves along the shock train. By determining the location of each measurement plane relative to the stationary shock train structure as seen in a schlieren image, we can infer the local structure of the flow along the shock train. This approximate mapping of each individual instantaneous measurement is constructed given the measured angle of the leading shock in the lambda foot $\left(\alpha_{L}\right)$ from schlieren and the wall-normal height of the core flow measured in each SPIV measurement (i.e., the portion of the flow above the lambda foot). Since $\alpha_{L}$ is nominally constant in time (see section III B) we can consistently determine the instantaneous location of each measurement plane relative to the stationary shock train. As a representative example of our SPIV measurements, we will present instantaneous velocity fields at four different measurement planes in the fixed shock train reference frame. The approximate streamwise locations and wall-normal height of the four SPIV measurement planes are indicated by the colored lines in figure 20. For convenience we will refer to the four measurement planes as $a, b, c$, and $d$ (see labels in figure 20).

The instantaneous streamwise velocity fields for measurement planes $a, b, c$, and $d$ are shown in figures 21(a), 21(b), 21(c), and 21(d), respectively. Measurement plane $a$, shown in figure 21(a), is located near the foot of the leading shock. The magenta contour line in the velocity field indicates the boundary of the core flow (labeled region I in the figure) that has not been processed by a shock wave (i.e., $U=0.99 U_{\infty}$ ). Region II in the figure is the flow that has passed through the leading shock of the lambda foot. The black contour line indicates the extent of the low-speed boundary layer, defined as the region where $U<250$ $\mathrm{m} / \mathrm{s}$. Note that the boundary layers (region III in the figure) have already thickened significantly relative to the undisturbed flow (compare to figure 19). On the side-wall, there are small regions of reversed flow (i.e, boundary layer separation), indicated by the dark blue color. No flow reversal occurs in the bottom-wall boundary layer. Measurement plane $b$, shown in figure 21(b), is located slightly further downstream, under the leading shock in the lambda foot. The size of the core flow (region I) is reduced because more of the flow has passed through the lambda foot leading shock (region II). Measurement plane $c$, shown in figure 21(c), is located just upstream of the leading shock Mach stem. At this point, all of the flow has passed through the leading shock in the lambda foot and thus the streamwise velocity throughout the entire field of view is less than $U_{\infty}$. Also, notice the development of a high-speed region of flow that reaches into the corner of the duct (see label $H$ ). Finally, measurement plane $d$, shown in figure 21(d), is located under the trailing shock of the lambda foot. In this instance, a portion of the flow (labeled region IV in the figure) has passed through both legs of the lambda shock, producing a region with an average streamwise velocity of $370 \mathrm{~m} / \mathrm{s}$. The magenta contour line indicates the boundary of the flow with a streamwise velocity of $U=370 \mathrm{~m} / \mathrm{s}$. A high-speed corner flow region is also evident in this measurement plane (see label $H$ ). Moving downstream from measurement plane $a$ to measurement plane $b$, the low-speed boundary layer (region III in the figures) grows and more separation is evident. By measurement plane $d$, the side-wall separation is approximately $13 \mathrm{~mm}$ (or $23 \%$ of the tunnel width) in thickness.

The instantaneous transverse velocity fields for measurement planes $a-d$ are shown in figures $22(\mathrm{a})-22(\mathrm{~d})$, 
respectively. Also, consider the instantaneous vertical velocity fields for measurement planes $a-d$ shown in figures 23(a)-23(d), respectively. In the core flow (region I enclosed by the magenta contour lines) the transverse and vertical velocity components are approximately zero. Similarly, the flow that has passed through both legs of the lambda foot (region IV in measurement plane $d$ ) has average $V$ and $W$ velocity components of $0 \mathrm{~m} / \mathrm{s}$. In all four measurement planes, the flow processed by the leading shock in the lambda foot (region II) is pushed upwards and towards the center of the duct, inducing positive $V$ and $W$ velocities. The low-speed boundary layers (region III) show mixed positive and negative $V$ and $W$ velocities.

Next, we will consider the separation regions in more detail. Recall that the four example instantaneous SPIV planes in figure 21 show significant boundary layer separation, indicated by the dark blue reversed flow regions. Figure 24 illustrates the probability of separation in the SPIV field of view. The probability map is calculated using all 400 instantaneous SPIV images, thus it describes the likelihood of separation between the leading shock foot and the most downstream measurement plane (measurement plane $d$ ). Separation is likely to occur on the side-wall, with the most probable separation region at $z=15 \mathrm{~mm}$. No separation is evident on the bottom-wall.

We determine the total amount of separation in each instantaneous SPIV image by quantifying the area over which there are negative streamwise velocities. In a similar manner, the size of the core flow is defined as the area over which the streamwise velocity is above $0.99 U_{\infty}$. Figure 25 illustrates a linear relationship between the separation area and the core area. When the core area is large (i.e., the measurement plane is near the leading shock foot) the separation area is approximately zero. The separation area increases linearly as the measurement plane moves downstream of the leading shock foot. Note that after the entirety of the flow in the field of view has been processed by the leading shock in the lambda foot we can no longer easily distinguish different (relative) measurements locations because the core flow is no longer visible. Thus, this linear relationship is only valid for measurement planes located at or upstream of measurement plane $c$ in figure 20 .

Flow streamlines are computed from the instantaneous velocity measurements to develop a better qualitative understanding of the shock and flow structure. Figures 26(a)-26(d) show the in-plane streamlines for measurement planes $a-d$, respectively, corresponding to the instantaneous velocity fields shown in figures 21-23. The core flow region is indicated by the green shaded area, while the regions of separated flow are indicated by the purple shaded area. In all four measurement planes, it is clear that the flow processed through the lambda foot converges towards the core flow. There is a degree of radial symmetry that indicates the shock structure may be axisymmetric.

Part of the leading shock structure is determined by evaluating the core flow (i.e., where $U=0.99 U_{\infty}$ ) contour shape in multiple measurement planes. First, the location of each individual SPIV image is found relative to the stationary shock. Then, the core flow contour of each measurement plane is evaluated for the region of flow under the leading shock lambda foot. Five contour lines from different instantaneous measurement planes at approximately the same relative location are averaged to determine the typical shape of the core flow at that location. The averaging process is repeated along the length of the leading shock in the shock train. Figure 27 shows the isosurface of the core flow found using the average contours. The isosurface describes part of the leading shock structure along all three coordinate directions for a stationary shock train. Too few measurements were collected downstream of the leading shock Mach stem to generate a representation of the core flow shape. Therefore, what shown in figure 27 only reflects measurement planes upstream of the Mach stem. In addition, SPIV measurements were only collected in one corner of the duct cross-section. Given the symmetry of the wind tunnel nozzle and test section across the $y$-centerline of the duct $(y=W / 2)$, the SPIV results are mirrored to get a complete qualitative view of the lower half of the test section.

Figure 27(a) is a planar view of the isosurface (as if the viewer is looking up the test section). The dashed line indicates the plane of symmetry $(y=W / 2)$ and the color of the isosurface corresponds to the distance from the leading shock foot. The side-wall separation isosurface is also included in figure 27(a) for reference and was calculated in the same manner as described above but instead using $U=0 \mathrm{~m} / \mathrm{s}$ contour lines. From this view it is clear that the area of the separated region grows and the core area shrinks as the measurement plane is moved downstream of the stationary leading shock foot. There is no bottom-wall separation isosurface because the bottom-wall has separated flow in less than $5 \%$ of the measurements.

Figure 27(b) is a perspective view of the core flow isosurface and demonstrates the three-dimensionality of the shock structure. The separation isosurface is omitted for clarity. The shock structure resembles that of a cone. This conical structure explains why the side- and bottom-wall pressure profiles begin to rise at 
approximately the same $x$-location (see section III B). From the two-dimensional projected structure of the shock train seen in a schlieren image, we would expect the bottom-wall pressure profile to rise prior to the side-wall pressure profile due to the significant lambda foot that reaches upstream of the Mach stem. What is not visible in a schlieren image is the large side-wall separated area that significantly confines the flow, especially near the corner. The thick boundary layers displace the shock structure away from both the sideand bottom-walls, resulting in the conical structure seen in figure 27(b).

Figure 28 is a model of the three-dimensional leading shock structure if the lambda foot conical shape is extrapolated to parts of the shock that were not measured with SPIV. In particular, the top half of the duct was not studied using SPIV so the conical shape is mirrored across the $z$-centerline plane of the duct $(z=H / 2)$. In addition, only a few measurement planes were captured downstream of the Mach stem (see measurement plane $d$ in the above figures). Nevertheless, a similar conical structure emerges from these limited number of examples. The overall shock structure resembles two truncated cones (frusta) with their small ends coinciding to form the Mach stem. Since the boundary layer continues to thicken, we expect the frustum downstream of the Mach stem to have a smaller base than the upstream frustum.

\section{Conclusions}

The current experiment simulates the flow phenomena in the isolator of a high-speed air-breathing engine. A back valve (analogous to a combustor) is used to control the downstream pressure that leads to the formation of a shock train in the test section (representative of an isolator). Inlet unstart will occur if the back pressure becomes too high. We consider cases before unstart where the back pressure is held constant. Despite the constant boundary conditions the shock train has an unsteady component and fluctuates about its time-average position. Schlieren movies and pressure measurements are used to determine the effects of changing back pressure on the shock train unsteadiness characteristics and structural properties. Stereoscopic particle image velocimetry (SPIV) is then used to study the structure of the leading shock in a normal shock train. A summary of our key results are as follows:

1. At a given back pressure, consecutive shocks in the shock train system have the same position fluctuation statistics (i.e., same average, maximum, and standard deviation) but downstream shocks tend to fluctuate faster. In addition, clear differences exist in the frequency content of the first four shocks. Downstream shocks exhibit high-frequency modes that indicate the unsteady dynamics change through the shock train system.

2. The leading shock fluctuation speed and magnitude of displacement are independent of back pressure. Thus, the unsteadiness dynamics do not depend on where the shock train is located.

3. Changes in the Mach stem height and angles of the lambda foot indicate that the leading shock of the shock train undergoes an oblique-to-normal transition process with increased back pressure. The timeaverage pressure profiles also indicate structural changes across the entire shock train. For example, the length of the shock train decreases and the pressure rise across the shock train increases as back pressure is increased (i.e., the shock train is pushed upstream).

4. SPIV measurements show the bottom-wall is not separated under the leading shock lambda foot. The side-wall has a large region of separation that grows along the length of the leading shock. Contours of the core flow from SPIV measurements show a degree of axisymmetry under the leading shock lambda foot that suggests a nominally conical shape. The overall leading shock structure resembles two truncated cones with the small ends coinciding to form the Mach stem.

\section{Acknowledgments}

RLH acknowledges the financial support of the National Science Foundation Graduate Research Fellowship Program under Grant No. DGE 1256260. The authors would like to thank the valuable help of Rohan Morajkar in conducting some of the experiments presented here. 


\section{References}

${ }^{1}$ Matsuo, K., Miyazato, Y., and Kim, H., "Shock train and pseudo-shock phenomena in internal gas flows," Progress in Aerospace Sciences, Vol. 35, No. 1, 1999, pp. 33-100.

${ }^{2}$ Sugiyama, H., Tsujiguchi, Y., and Honma, T., "Structure and oscillation phenomena of pseudo-shock waves in a straight square duct at Mach 2 and 4," 15th AIAA International Space Planes and Hypersonic Systems and Technologies Conference, AIAA paper 2008-2646, American Institute of Aeronautics and Astronautics, 2008.

${ }^{3}$ Lindstrom, C. D., Davis, D., Williams, S., and Tam, C., "Shock-train structure resolved with absorption spectroscopy part II: analysis and CFD comparison," AIAA Journal, Vol. 47, No. 10, 2009, pp. 2379-2390.

${ }^{4}$ Klomparens, R. L., Driscoll, J. F., and Gamba, M., "Unsteadiness characteristics and pressure distribution of an oblique shock train," AIAA Scitech, paper no. 2015-1519, 2015.

${ }^{5}$ Ikui, T., Matsuo, K., Nagai, M., and Honjo, M., "Oscillation phenomena of pseudo-shock waves," Bulletin of JSME, Vol. 17, No. 112, 1974, pp. 1278-1285.

${ }^{6}$ Nill, L. D. and Mattick, A. T., "An experimental study of shock structure in a normal shock train," AIAA 34th Aerospace Sciences Meeting and Exhibit, AIAA paper 96-0799, American Institute of Aeronautics and Astronautics, 1996.

${ }^{7}$ Wagner, J. L., Yuceil, K. B., Valdivia, A., Clemens, N. T., and Dolling, D. S., "Experimental investigation of unstart in an inlet/isolator model in Mach 5 flow," AIAA Journal, Vol. 47, No. 6, 2009, pp. 1528-1542.

${ }^{8}$ Rodi, P. E., Emami, S., and Trexler, C. A., "Unsteady pressure behavior in a ramjet/scramjet inlet," Journal of propulsion and power, Vol. 12, No. 3, 1996, pp. 486-493.

${ }^{9}$ Tan, H., Li, L., Wen, Y., and Zhang, Q., "Experimental investigation of the unstart process of a generic hypersonic inlet," AIAA Journal, Vol. 49, No. 2, 2011, pp. 279-288.

${ }^{10}$ Ikui, T., Matsuo, K., and Nagai, M., "The mechanism of pseudo-shock waves," Bulletin of JSME, Vol. 17, No. 108, 1974, pp. 731-739.

${ }^{11}$ Carroll, B. F. and Dutton, J. C., "Characteristics of multiple shock wave/turbulent boundary-layer interactions in rectangular ducts," Journal of Propulsion and Power, Vol. 6, No. 2, 1990, pp. 186-193.

${ }^{12}$ Geerts, J. S. and Yu, K. H., "Corner flow separation from shock train / turbulent boundary-layer interactions in rectangular isolators," 20th AIAA International Space Planes and Hypersonic Systems and Technologies Conference, paper no. 2015-3546, 2015.

${ }^{13}$ Le, D. B., Goyne, C. P., and Krauss, R. H., "Shock train leading-edge detection in a dual-mode scramjet," Journal of Propulsion and Power, Vol. 24, No. 5, 2008, pp. 1035-1041.

${ }^{14}$ Hutzel, J. R., Decker, D. D., Cobb, R. G., King, P. I., Veth, M. J., and Donbar, J. M., "Scramjet isolator shock train location techniques," 49th AIAA Aerospace Sciences Meeting including the New Horizons Forum and Aerospace Exposition, AIAA paper 2011-402, American Institute of Aeronautics and Astronautics, 2011.

${ }^{15}$ Sajben, M., Donovan, J. F., and Morris, M. J., "Experimental investigation of terminal shock sensors for mixedcompression inlets," Journal of Propulsion and Power, Vol. 8, No. 1, 1992, pp. 168-174.

${ }^{16}$ Handa, T., Masuda, M., and Matsuo, K., "Three-dimensional normal shock-wave/boundary-layer interaction in a rectangular duct," AIAA Journal, Vol. 43, No. 10, 2005, pp. 2182-2187.

$\checkmark{ }^{17}$ Do, H., Im, S., Mungal, M. G., and Cappelli, M. A., "The influence of boundary layers on supersonic inlet flow unstart induced by mass injection," Experiments in Fluids, Vol. 51, No. 3, 2011, pp. 679-691.

-18 Morgan, B., Duraisamy, K., and Lele, S. K., "Large-eddy and RANS simulations of a normal shock train in a constant-area isolator," 50th AIAA Aerospace Sciences Meeting, AIAA paper 2012-1094, 2012.

${ }^{19}$ Morgan, B., Duraisamy, K., and Lele, S. K., "Large-eddy simulations of a normal shock train in a constant-area isolator," AIAA Journal, Vol. 52, No. 3, 2014, pp. 539-558.

${ }^{20}$ Samimy, M. and Lele, S. K., "Motion of particles with inertia in a compressible free shear layer," Physics of Fluids, Vol. 3, No. 8, 1991, pp. 1915.

${ }^{21}$ Garcia, D., "Robust smoothing of gridded data in one and higher dimensions with missing values," Computational Statistics and Data Analysis, Vol. 54, 2010, pp. 1167-1178.

${ }^{22}$ Miyazato, Y., Matsuo, K., and Kasada, R., "Experimental and theoretical investigations of normal shock wave/turbulent boundary-layer interactions at low Mach numbers in a square straight duct," 47th AIAA Aerospace Sciences Meeting, AIAA paper 2009-925, 2009.

${ }^{23}$ Fotia, M. L. and Driscoll, J. F., "Isolator-combustor interactions in a direct-connect ramjet-scramjet experiment," Journal of Propulsion and Power, Vol. 28, No. 1, 2012, pp. 83-95. 


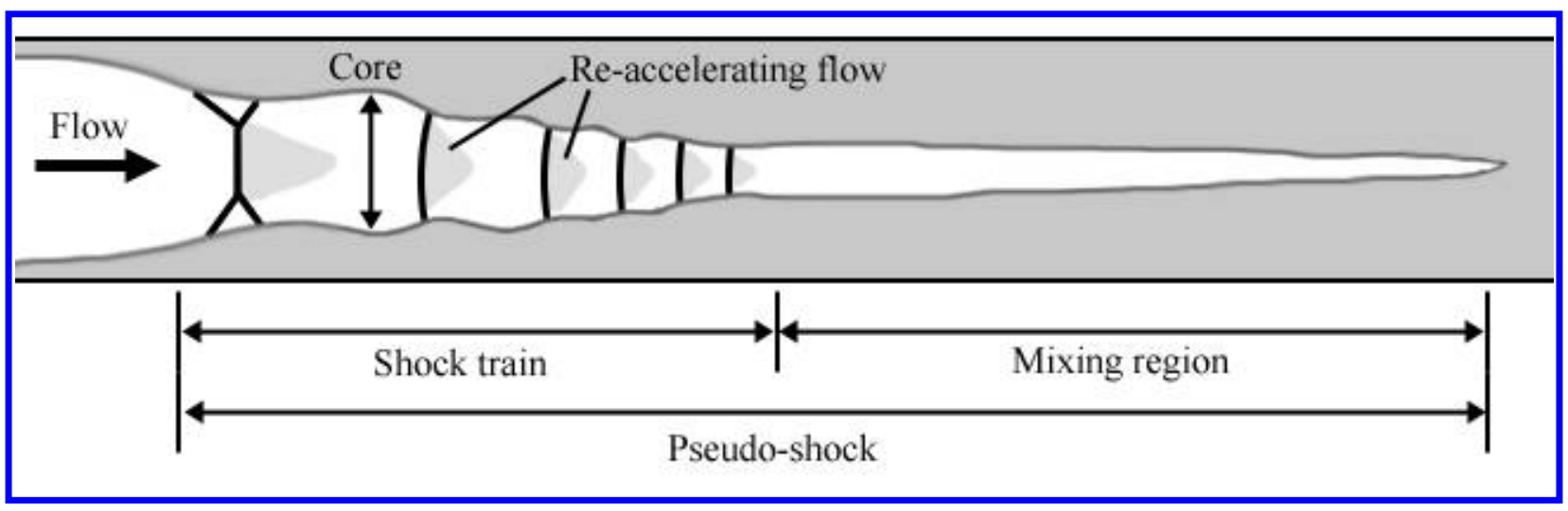

(a)

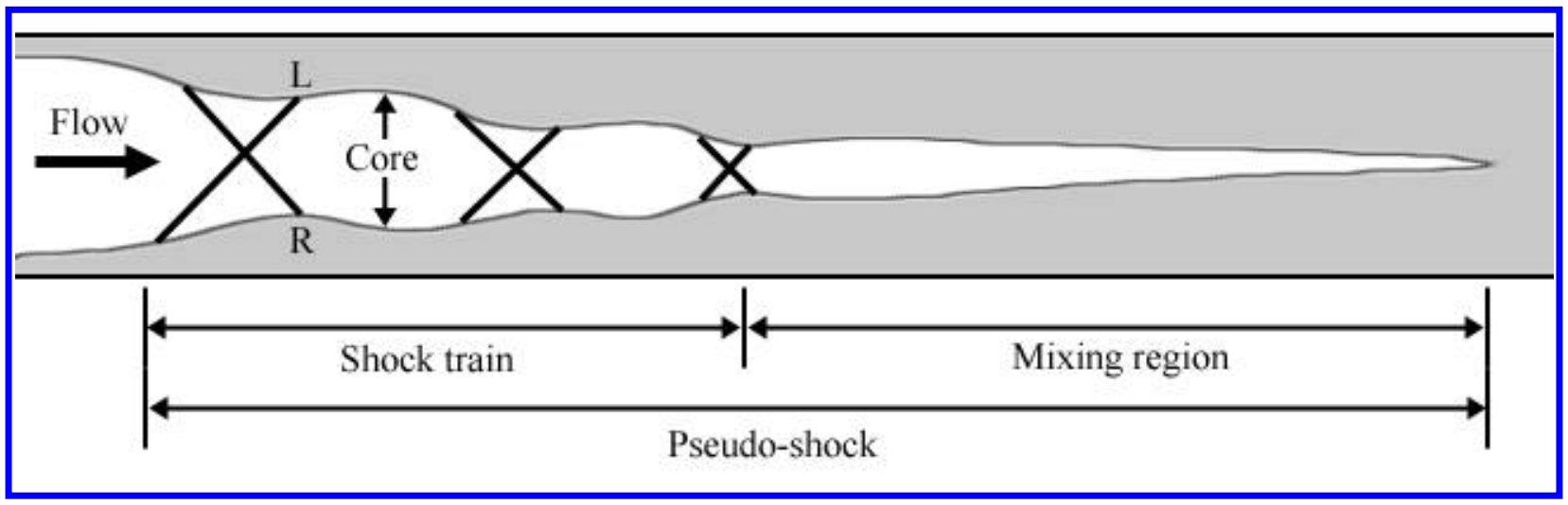

(b)

Figure 1. Schematic of a pseudo-shock adapted from Matsuo et al. (1999): (a) normal shock train; (b) oblique shock train.

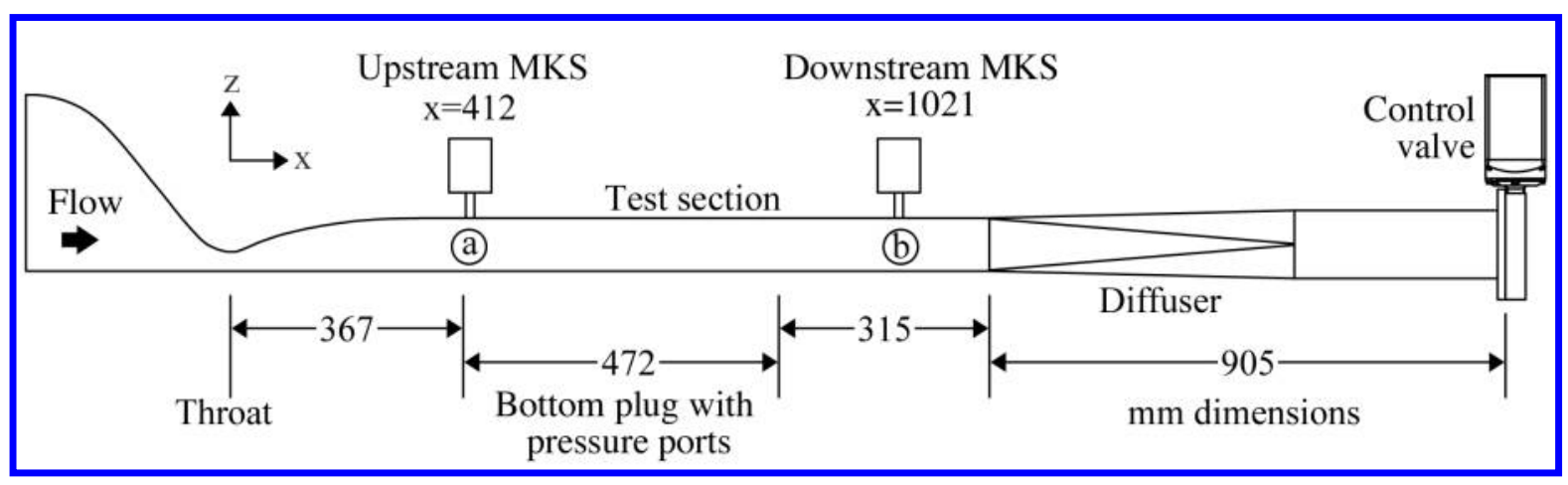

Figure 2. Schematic diagram of the wind tunnel (side view). 


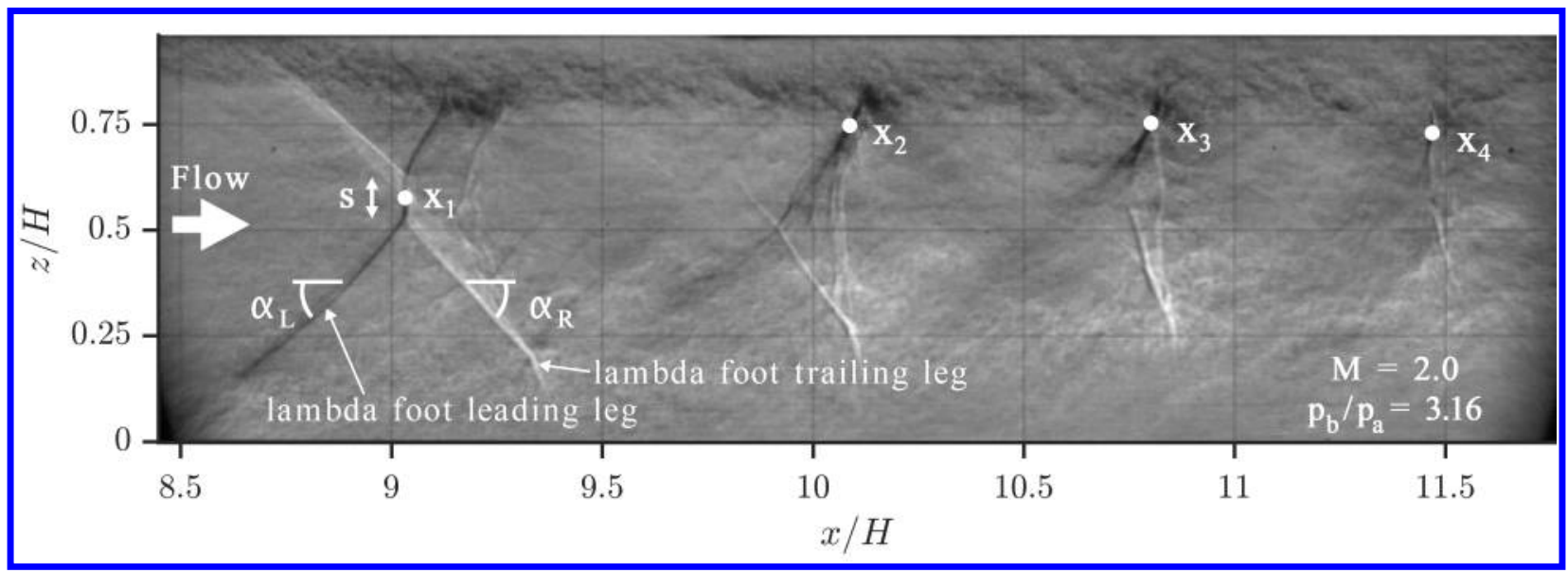

Figure 3. Instantaneous schlieren image of a Mach 2.0 shock train demonstrating tracked morphological features.

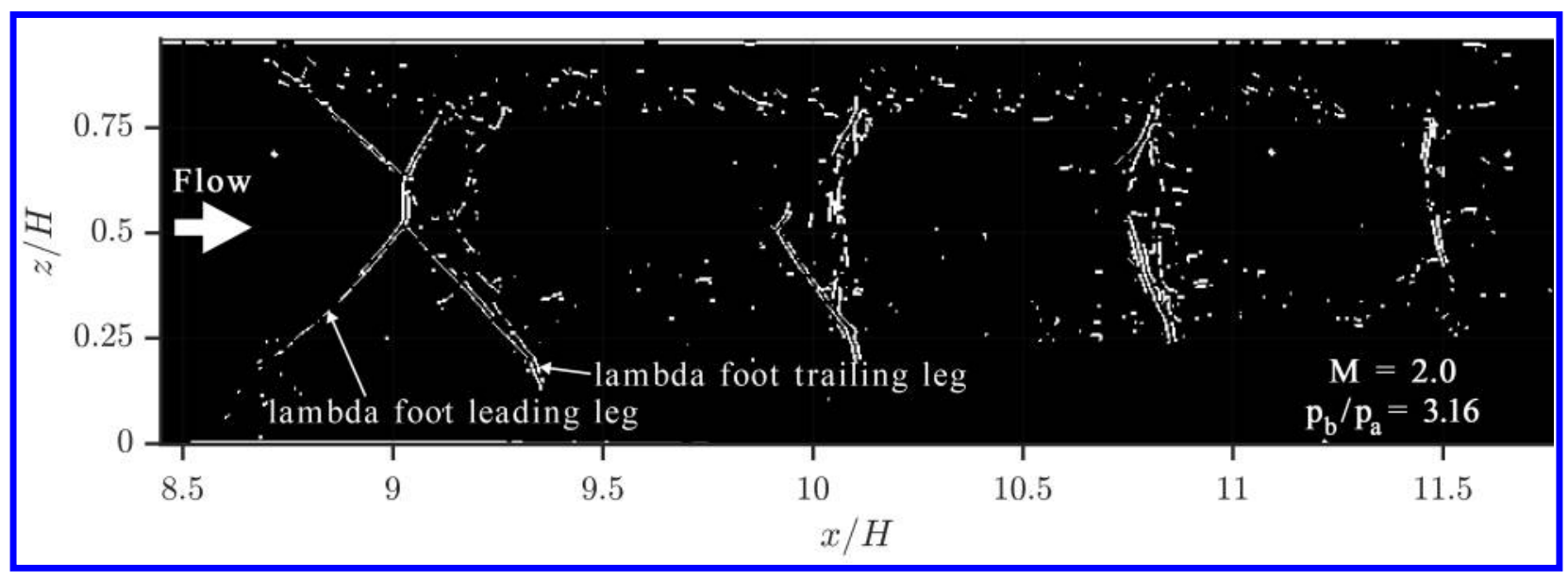

Figure 4. Instantaneous schlieren image with Sobel and median filters applied.

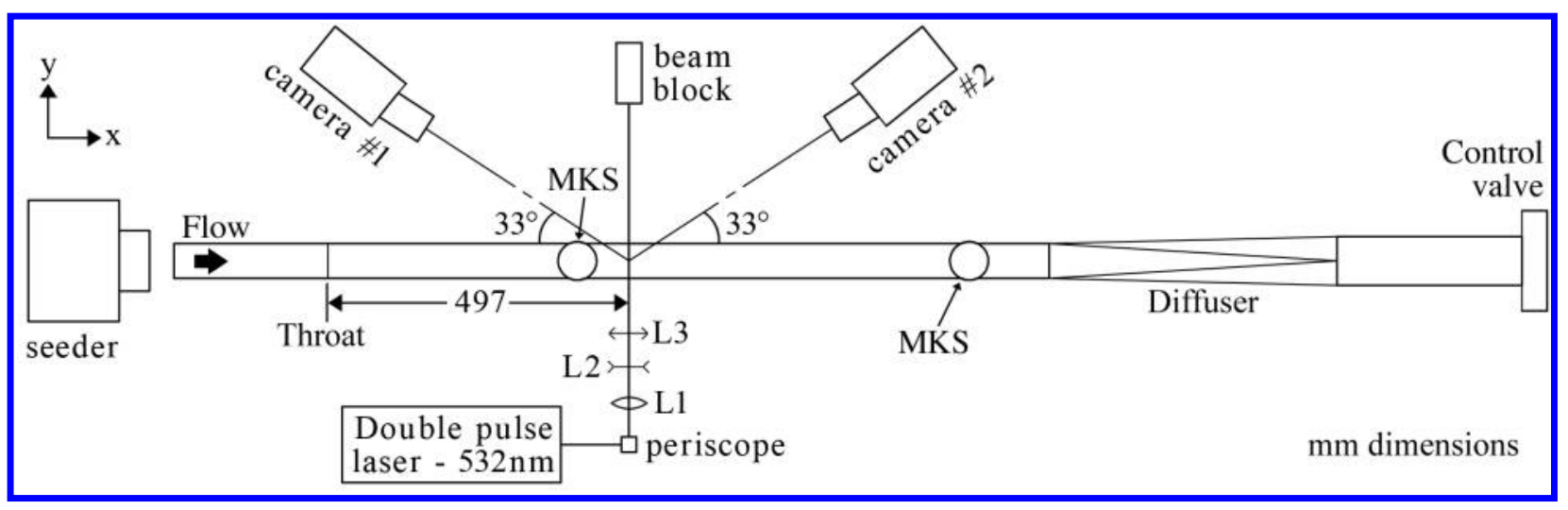

Figure 5. Schematic diagram of the stereo particle image velocimetry arrangement (top view). 


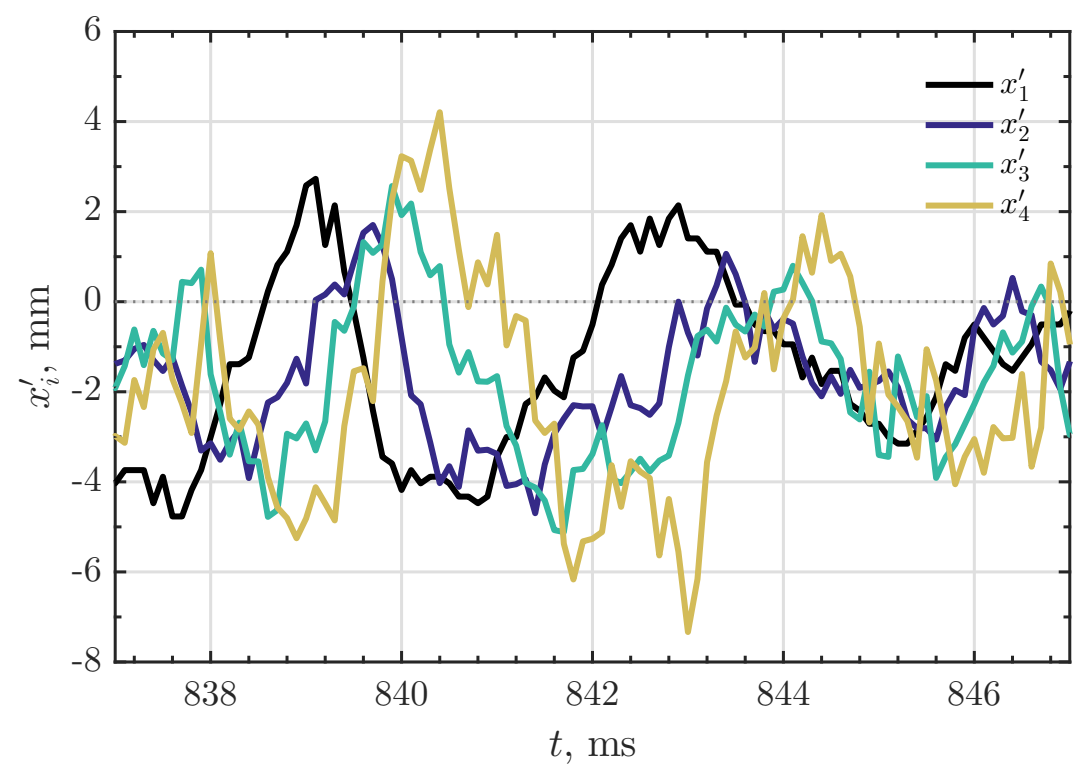

Figure 6. Time trace of shock position fluctuations.

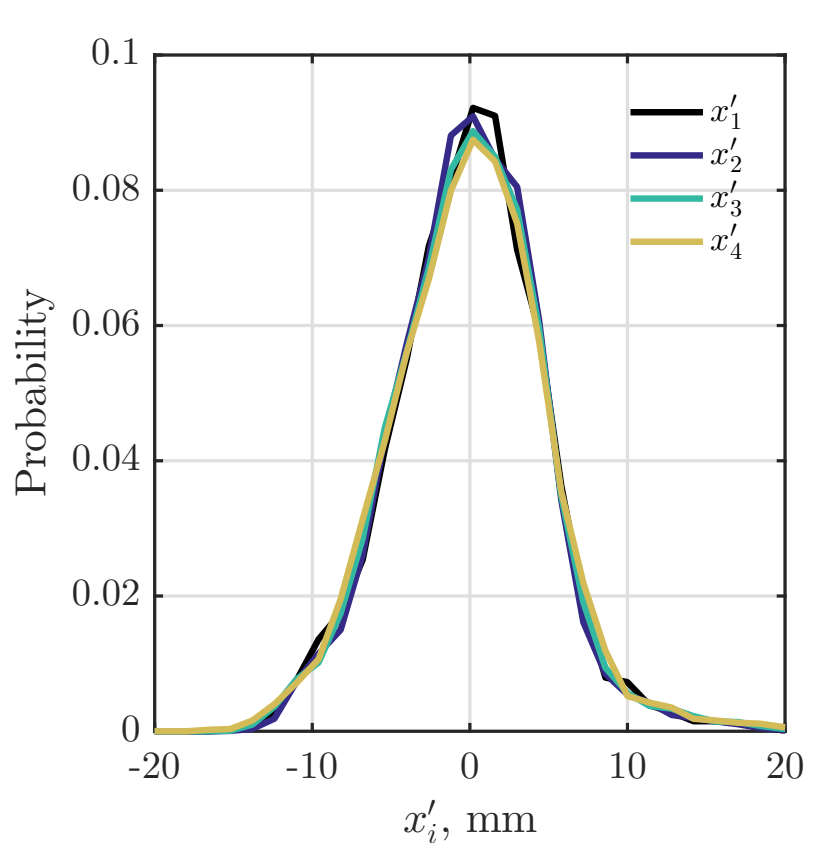

(a)

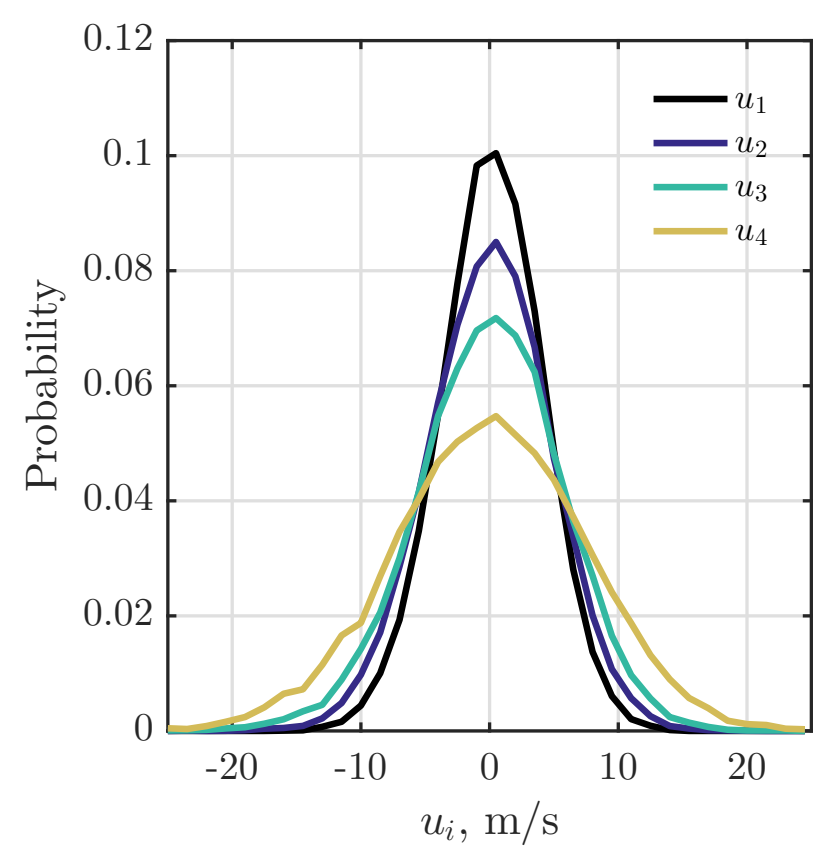

(b)

Figure 7. Probability density functions for the first four shocks of a Mach 2.0 shock train: (a) shock position fluctuation amplitude; (b) shock speed. 


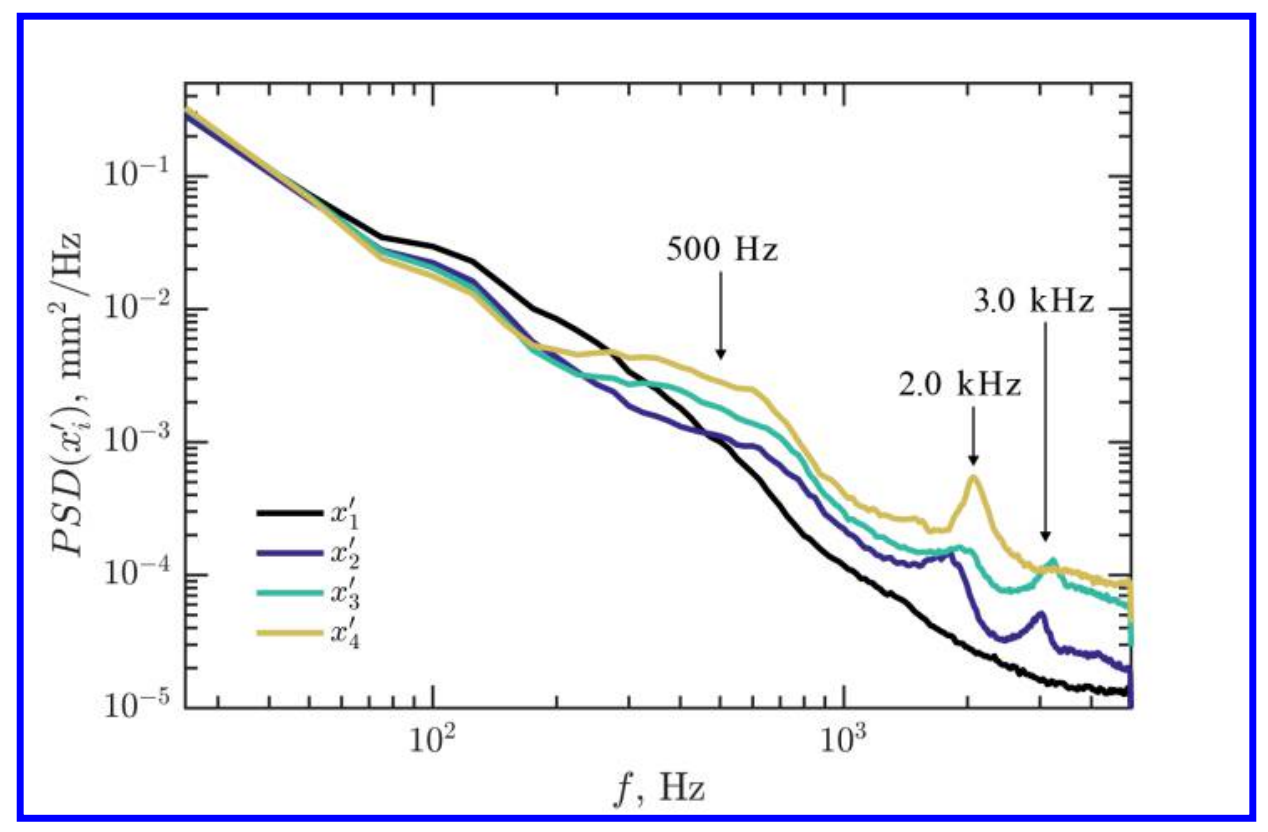

Figure 8. Power spectra of the shock position fluctuations.

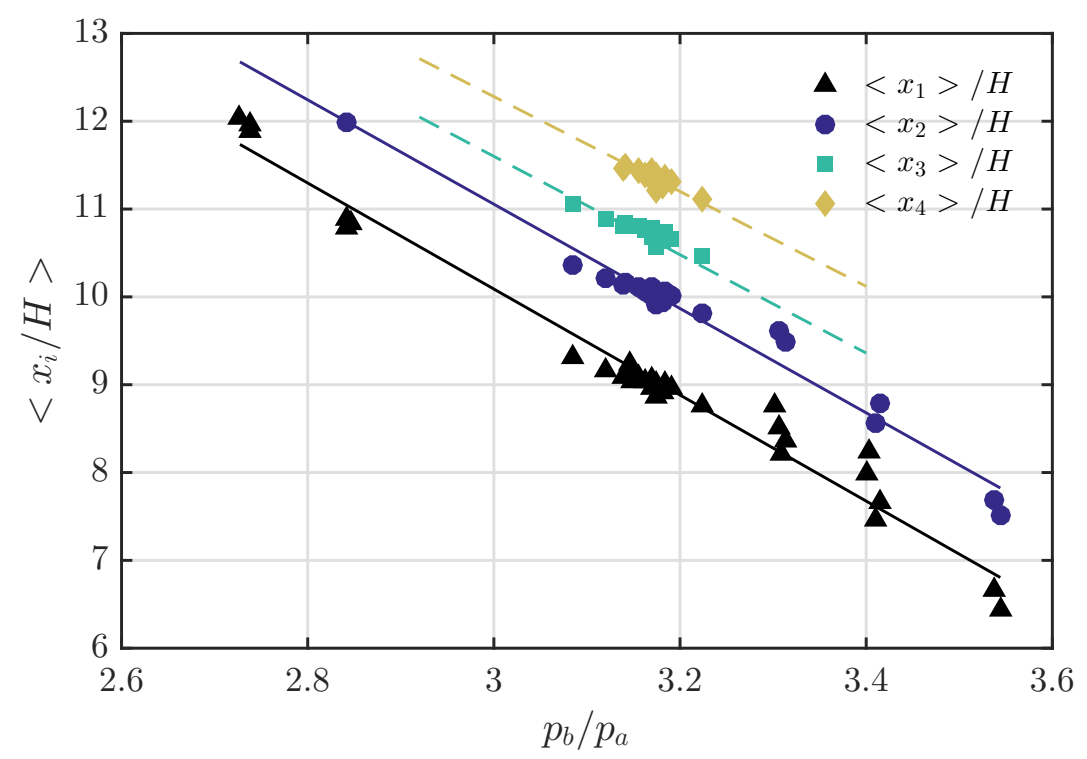

Figure 9. Average locations of the first four shocks as a function of back pressure. 


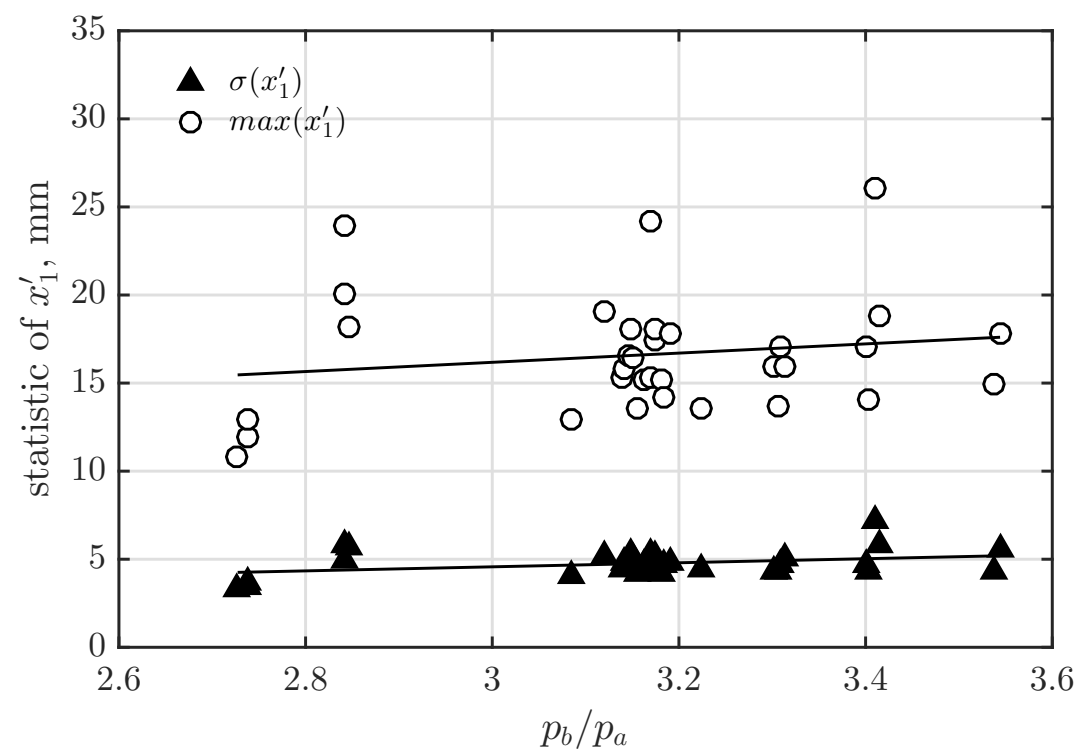

Figure 10. Maximum and standard deviation of shock fluctuation amplitude versus back pressure ratio.

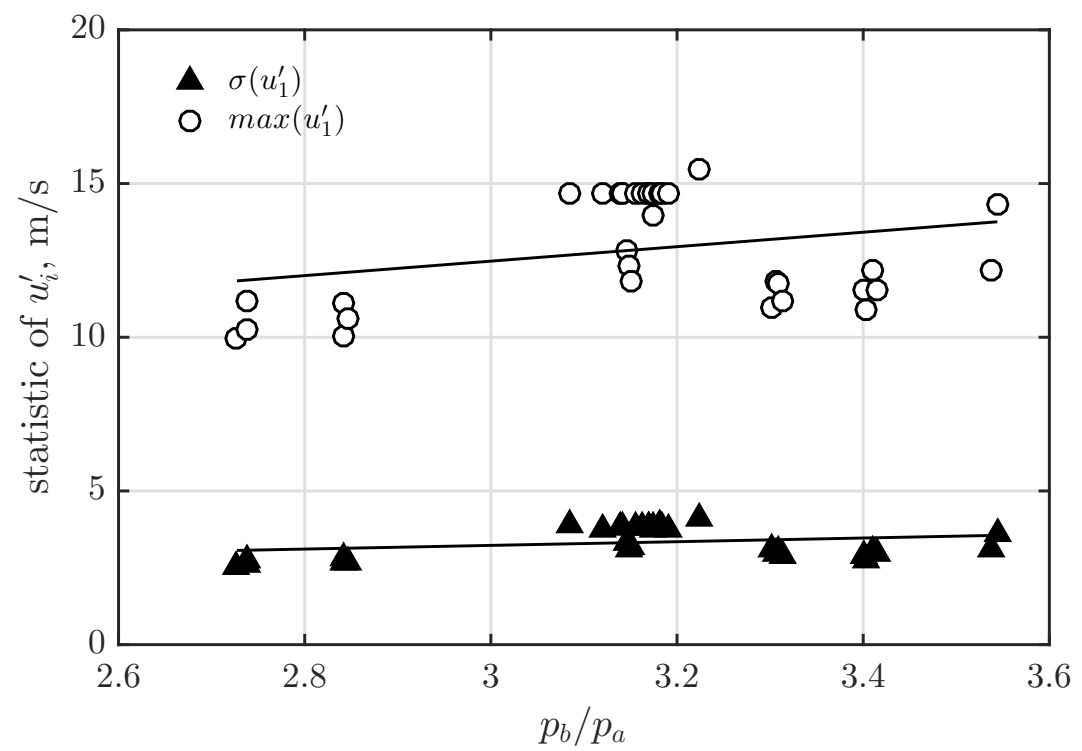

Figure 11. Maximum and standard deviation of shock speed versus back pressure ratio. 


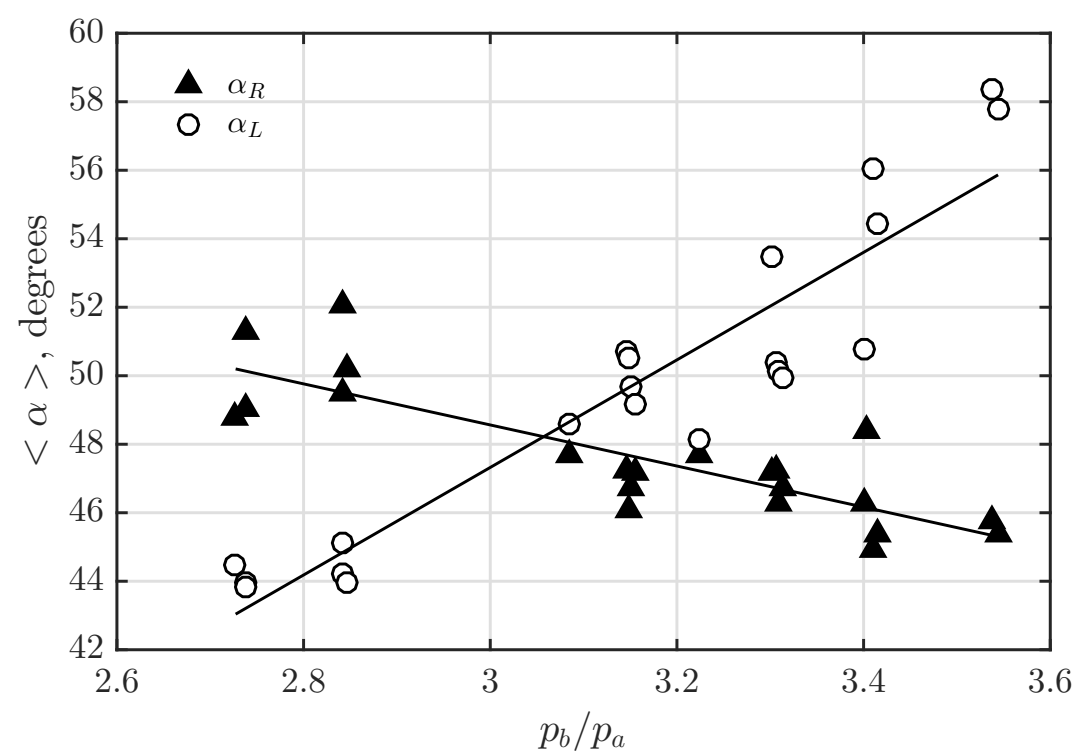

Figure 12. Time-average shock angles versus back pressure ratio.

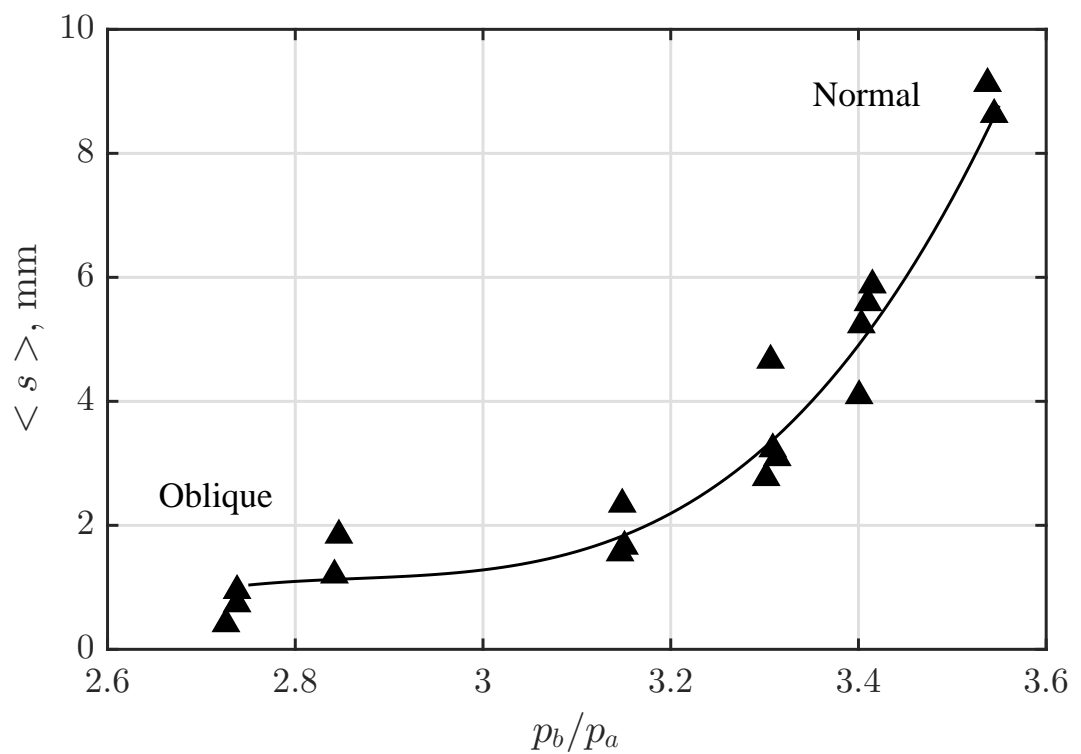

Figure 13. Time-averaged Mach stem height as a function of back pressure at $M=2.0$ 


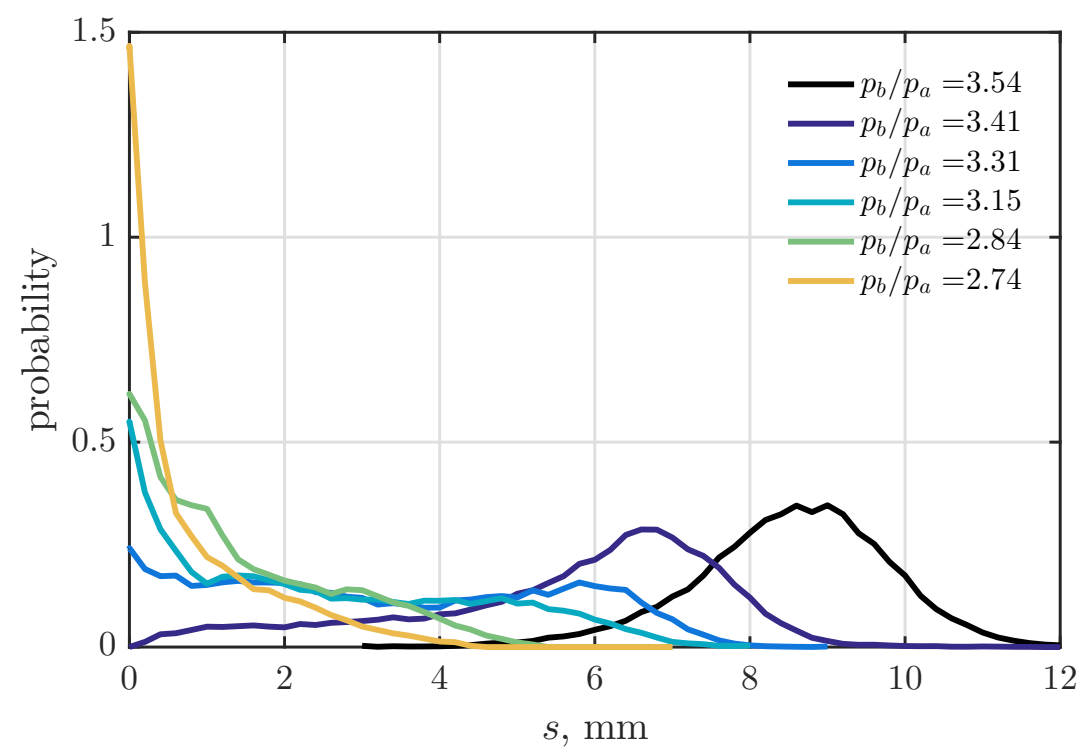

Figure 14. Probability density function of the Mach stem height at different back pressures at $M=2.0$.

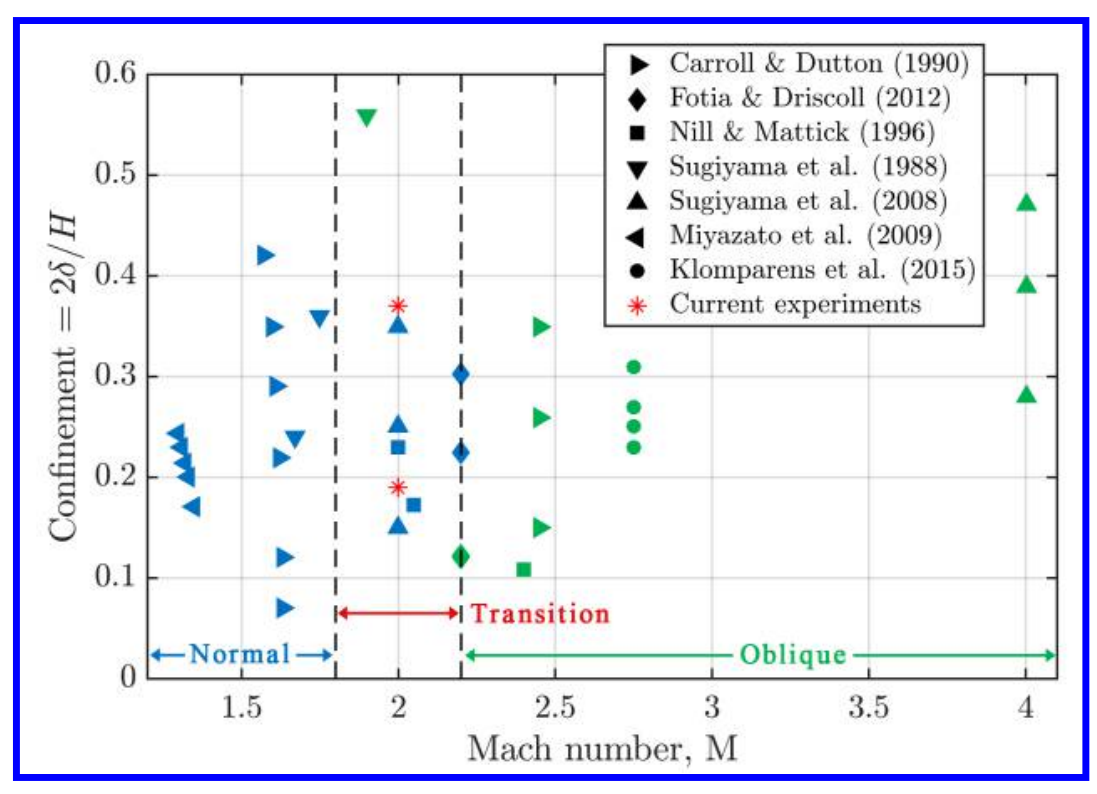

Figure 15. Shock train regime diagram. 


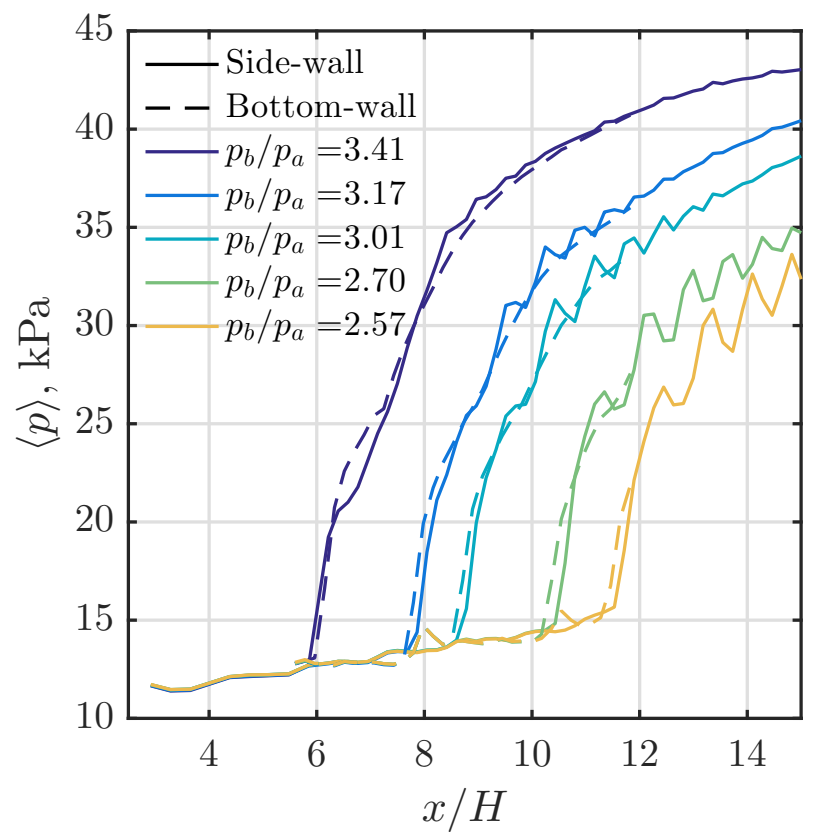

(a)

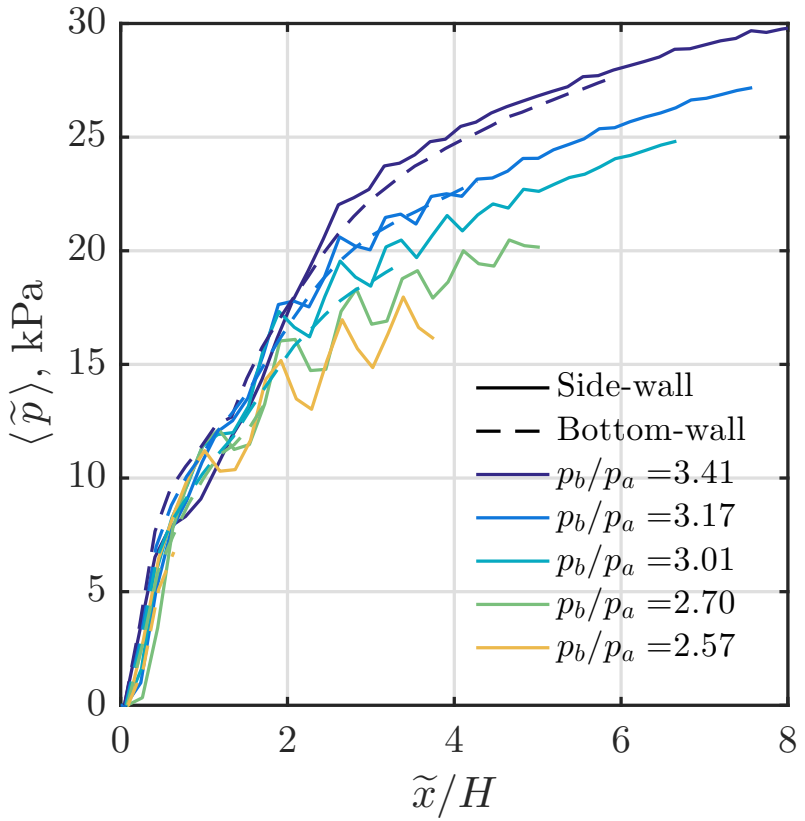

(b)

Figure 16. Time-average pressure profiles: (a) versus normalized location in the test section; (b) shifted profiles. 


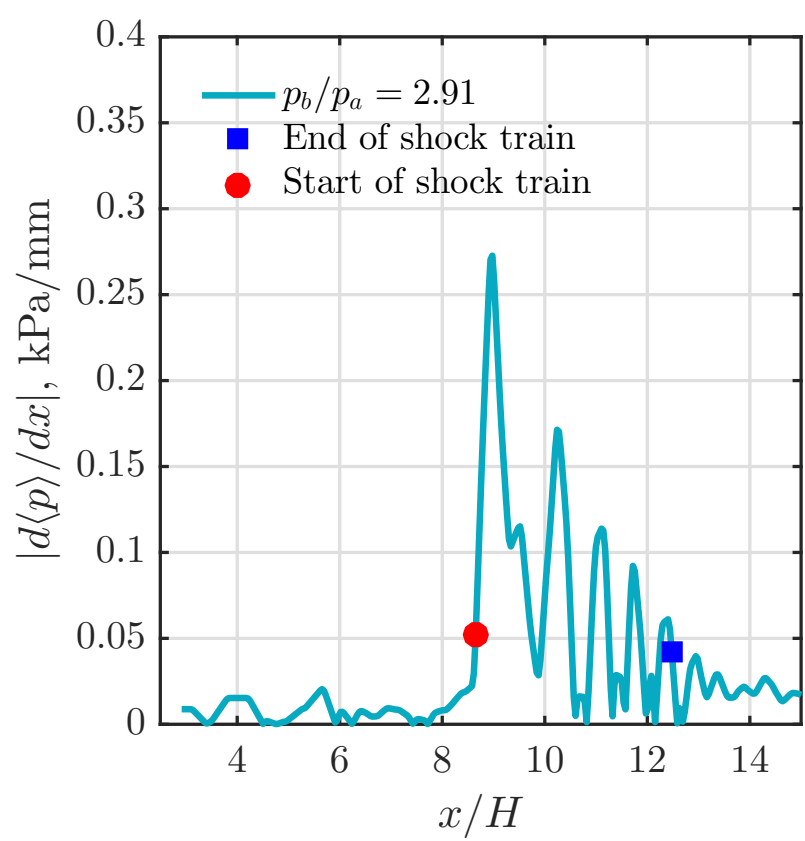

(a)

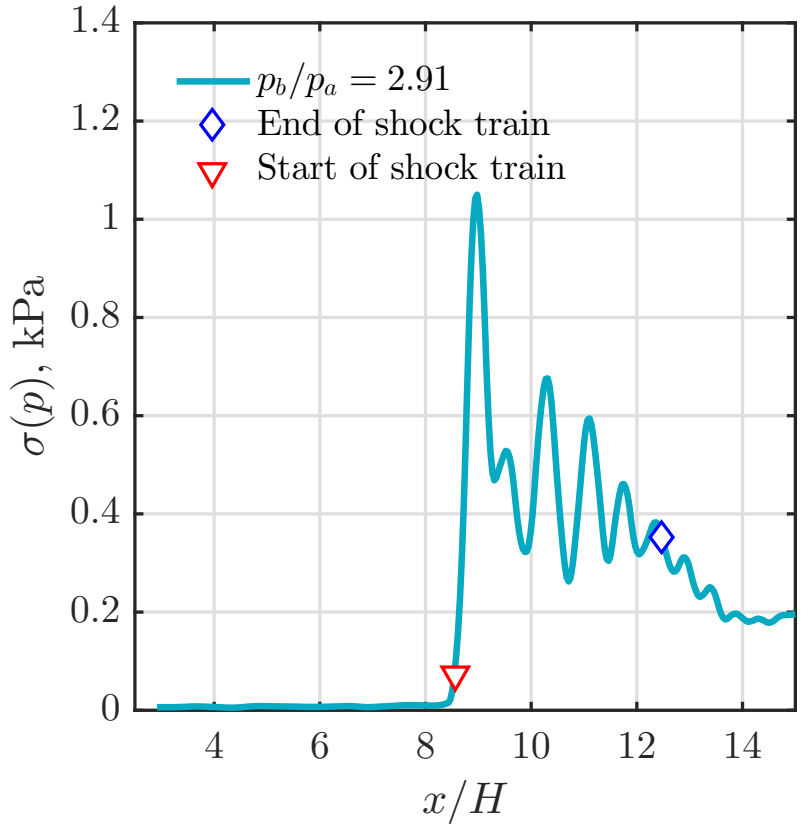

(b)

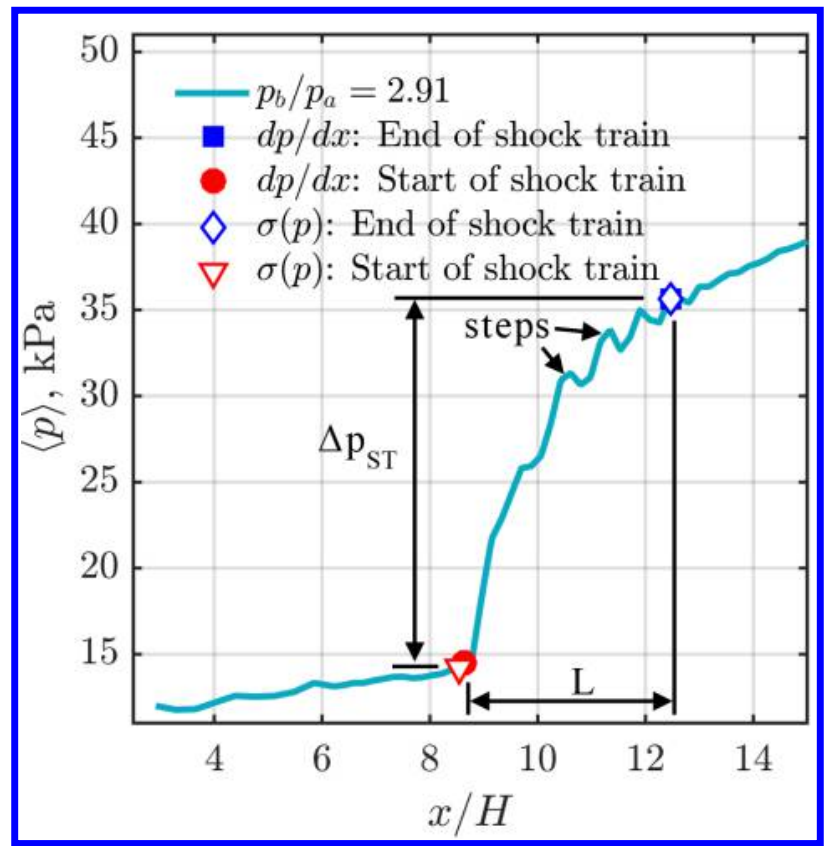

(c)

Figure 17. Example of how the shock train region is defined: (a) derivative of pressure with respect to $x$; (b) standard deviation of pressure; (c) pressure profile with beginning and end of shock train marked. 


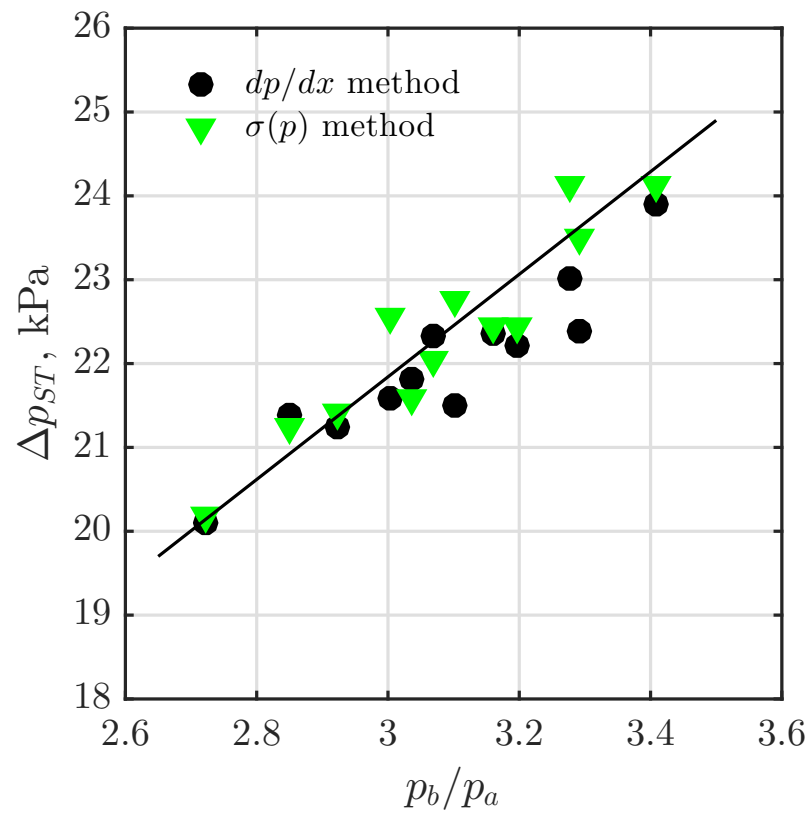

(a)

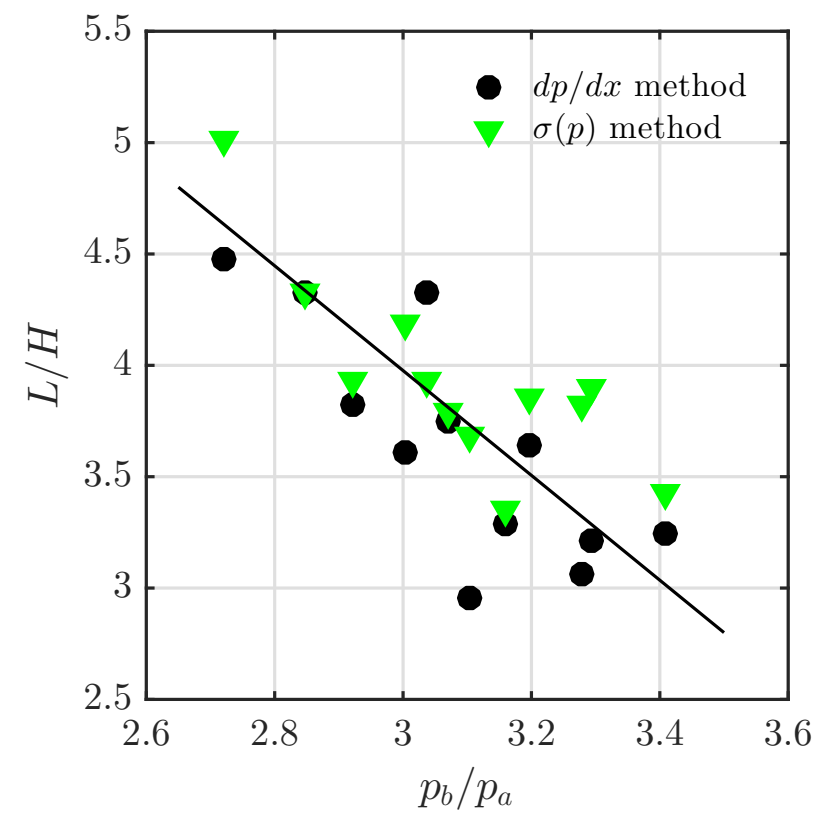

(b)

Figure 18. Shock train properties as a function of back pressure: (a) pressure rise across the shock train; (b) shock train length. 


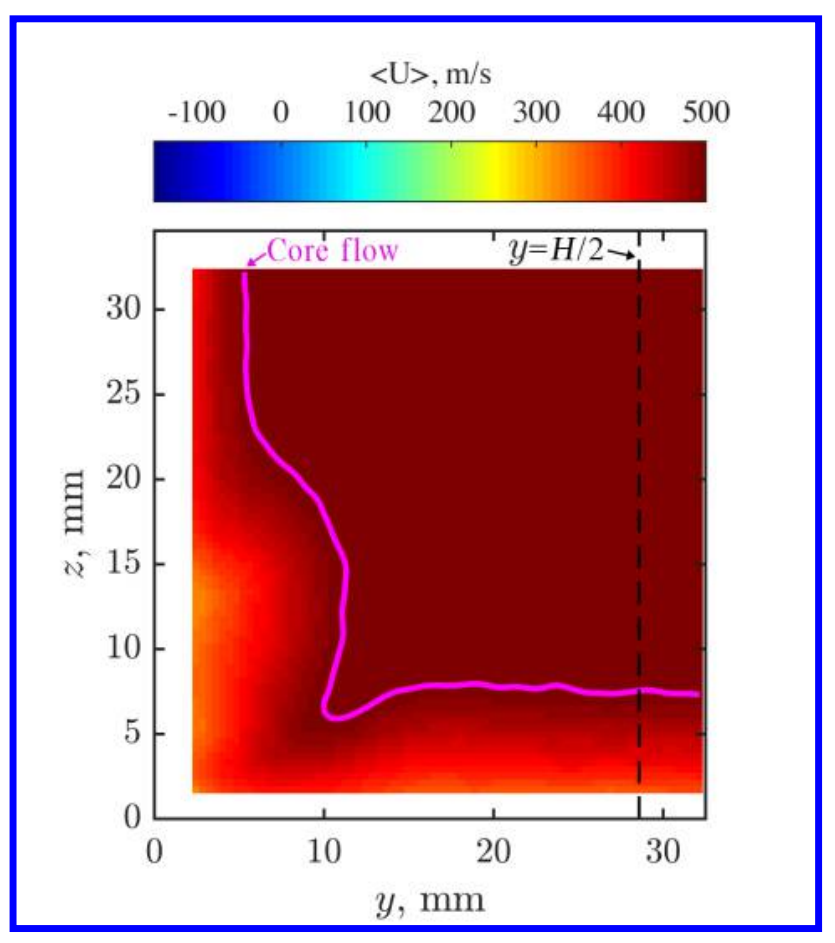

(a)

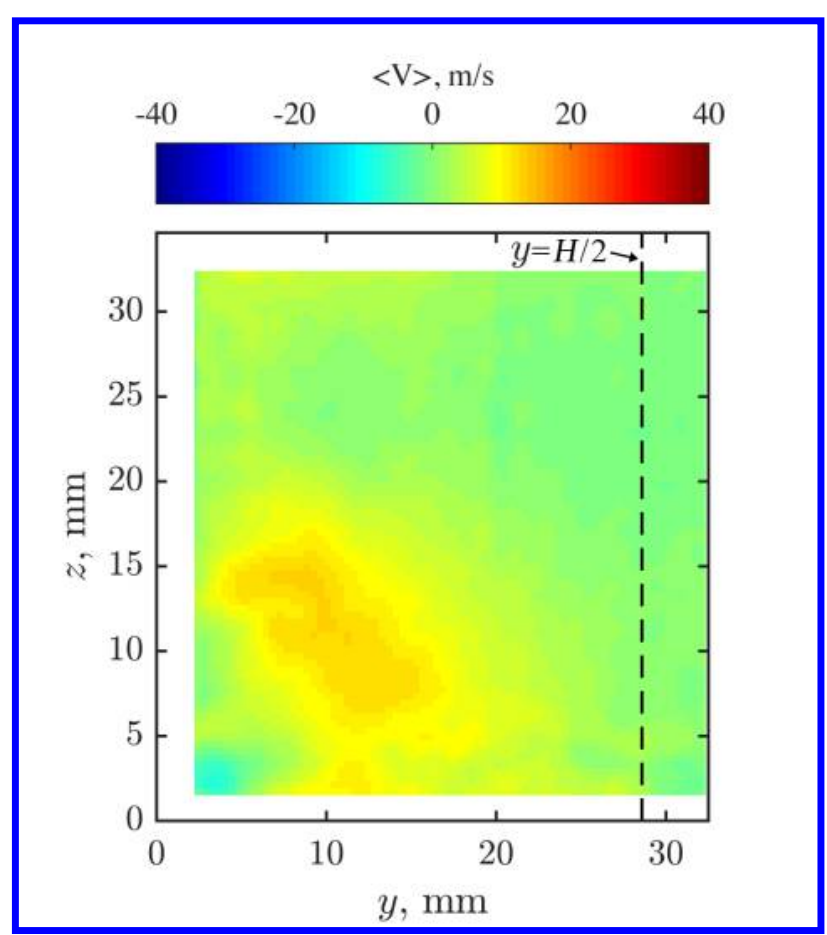

(b)

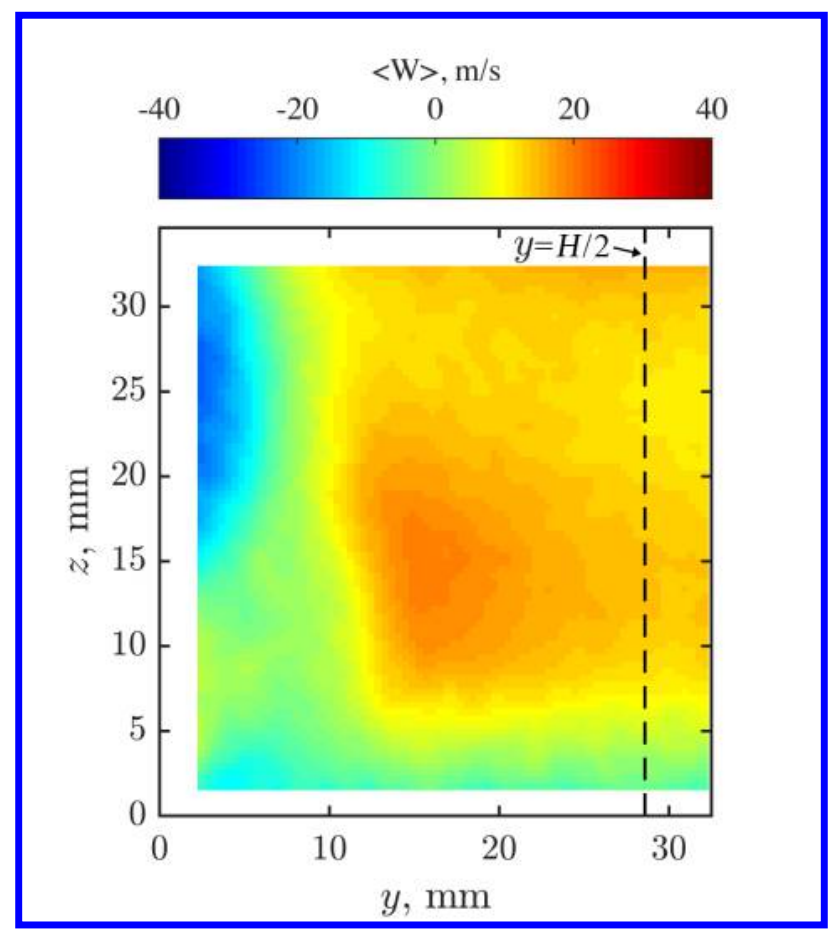

(c)

Figure 19. Time-average velocity fields without the presence of a shock train: (a) streamwise velocity, $U$; (b) transverse velocity, $V ;$ (c) vertical velocity, $W$. 


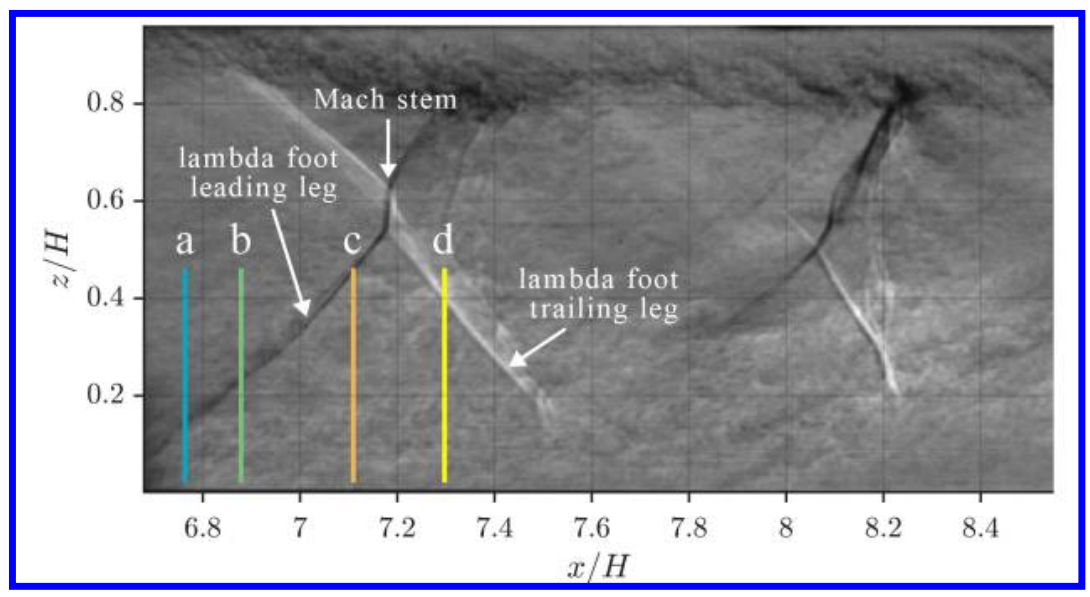

Figure 20. Schlieren image indicating where the four SPIV images in figure 21 are located in the shock train. 


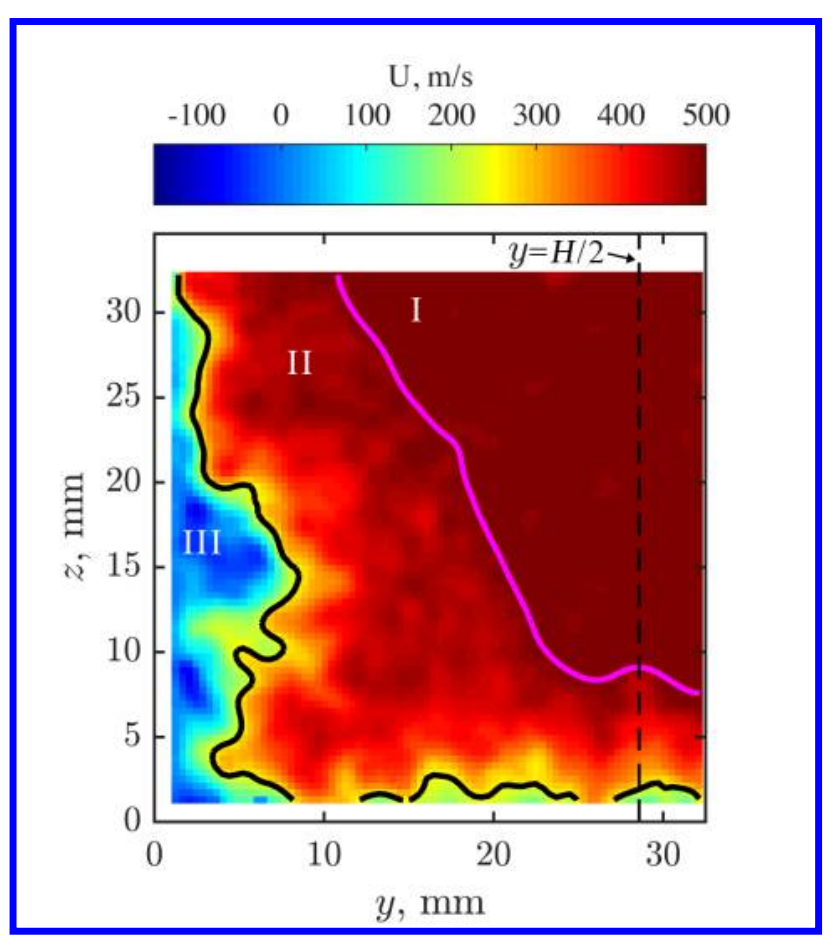

(a)

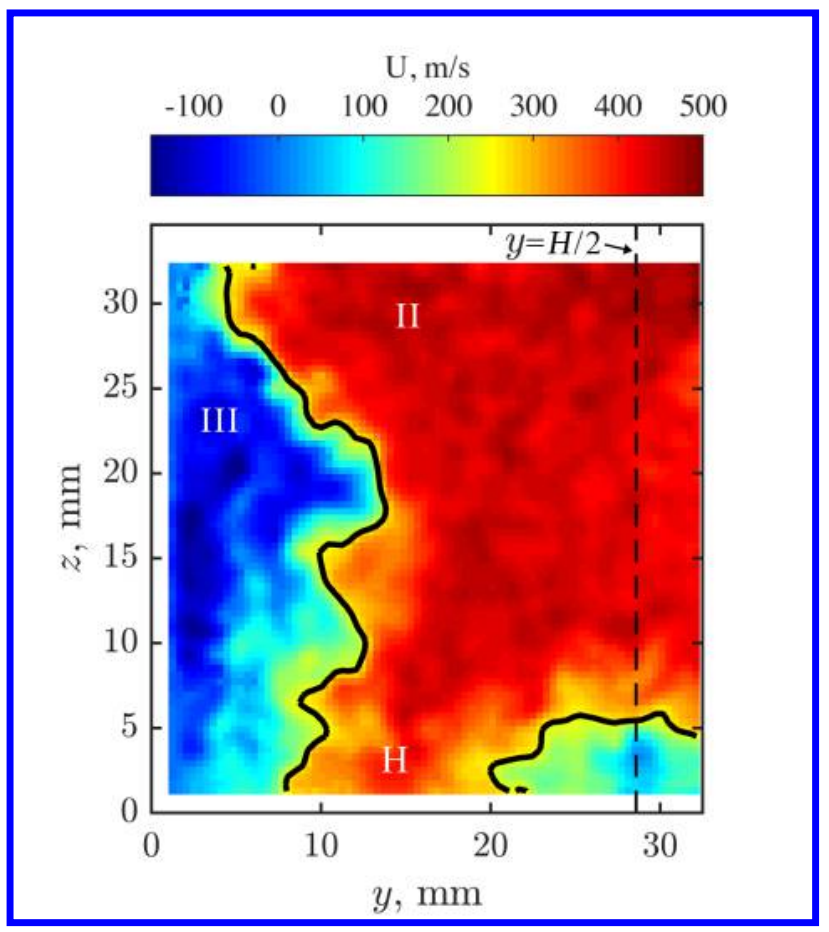

(c)

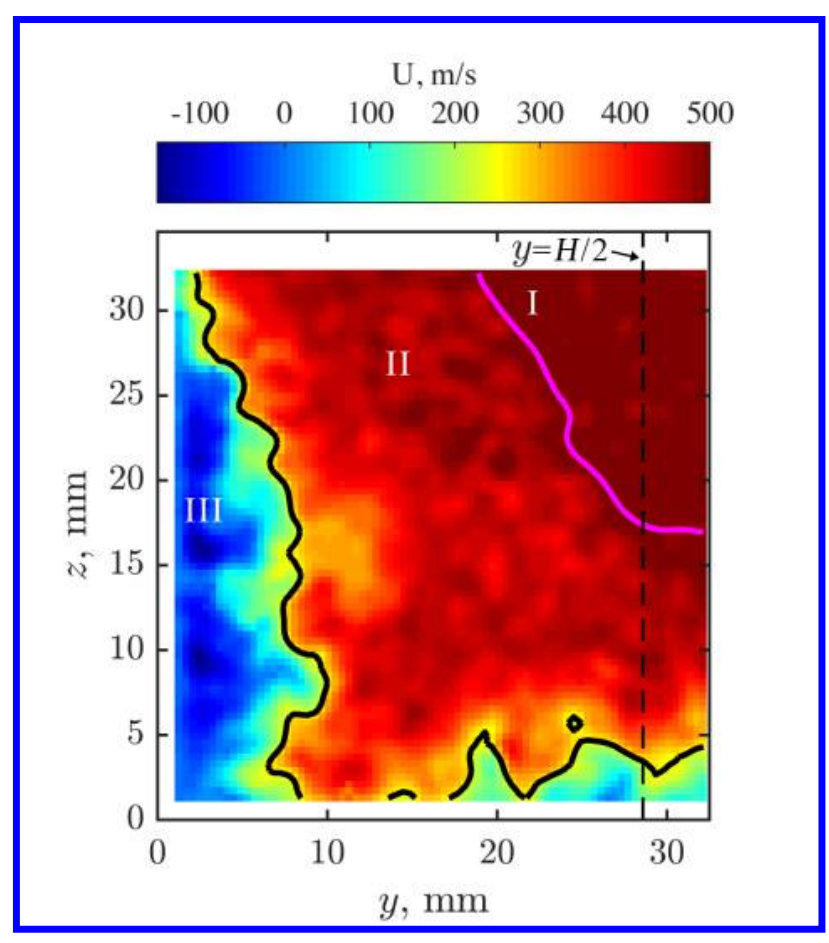

(b)

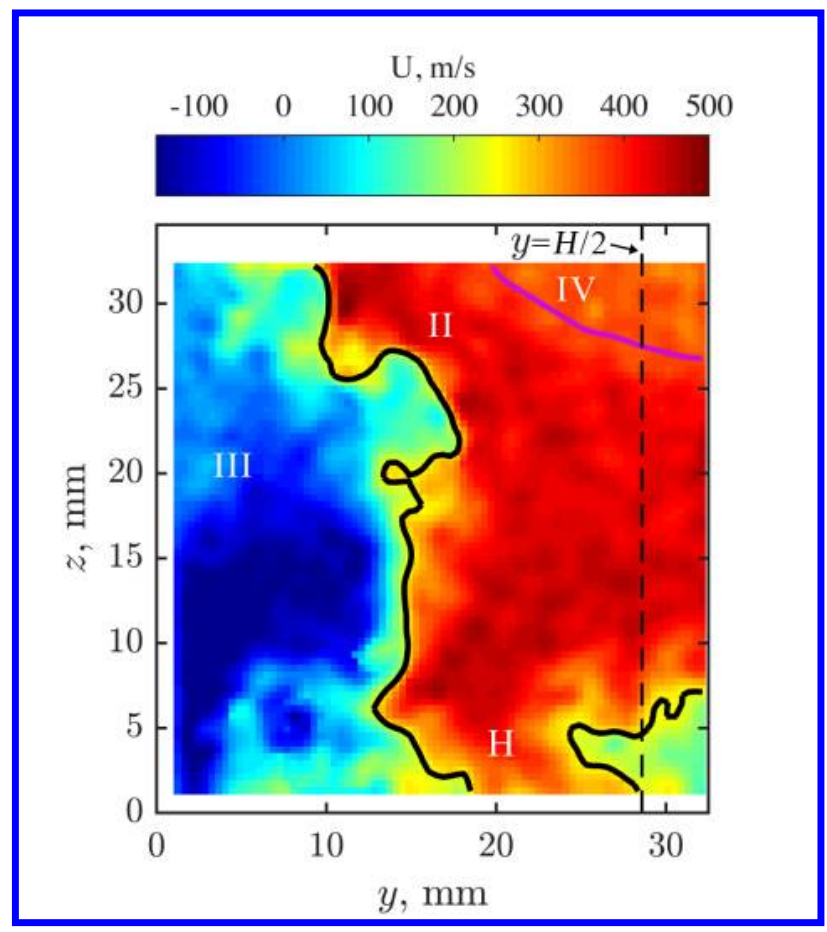

(d)

Figure 21. Instantaneous $U$ velocity field: (a) image plane $a$; (b) image plane $b$; (c) image plane $c$; (d) image plane $d$. 


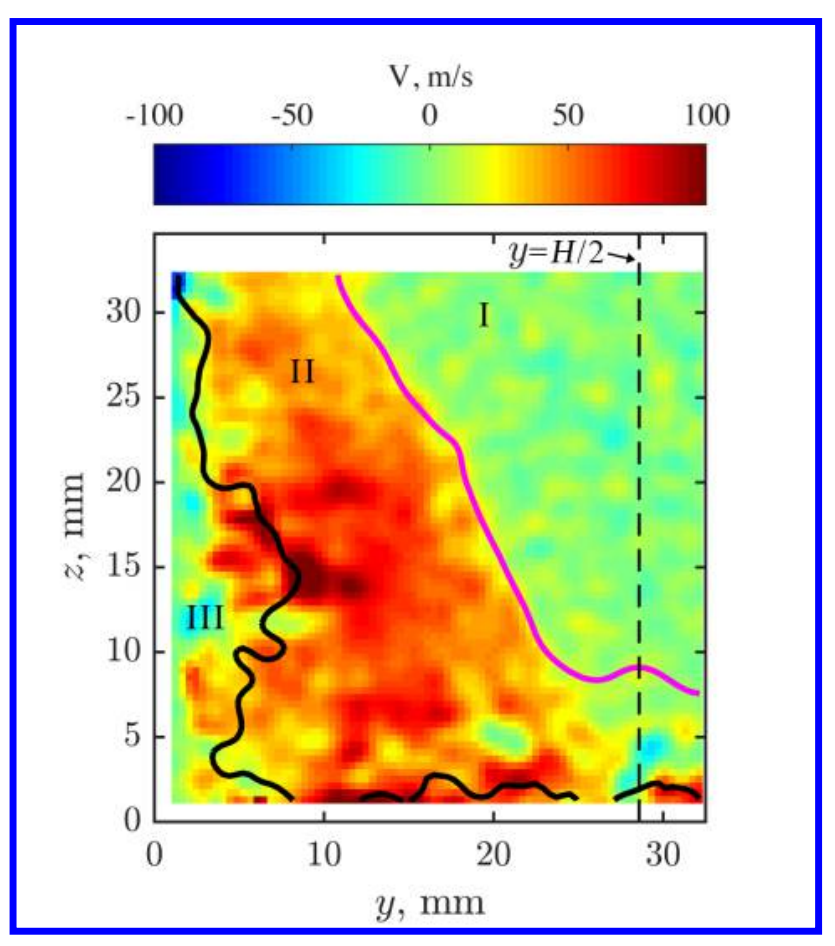

(a)

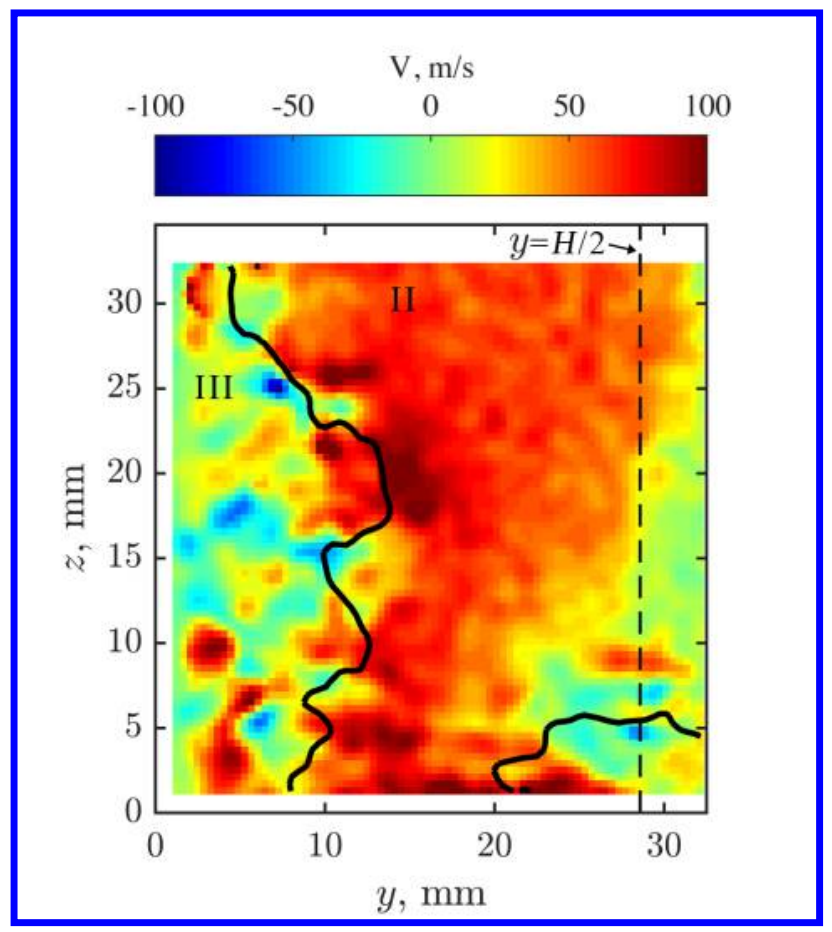

(c)

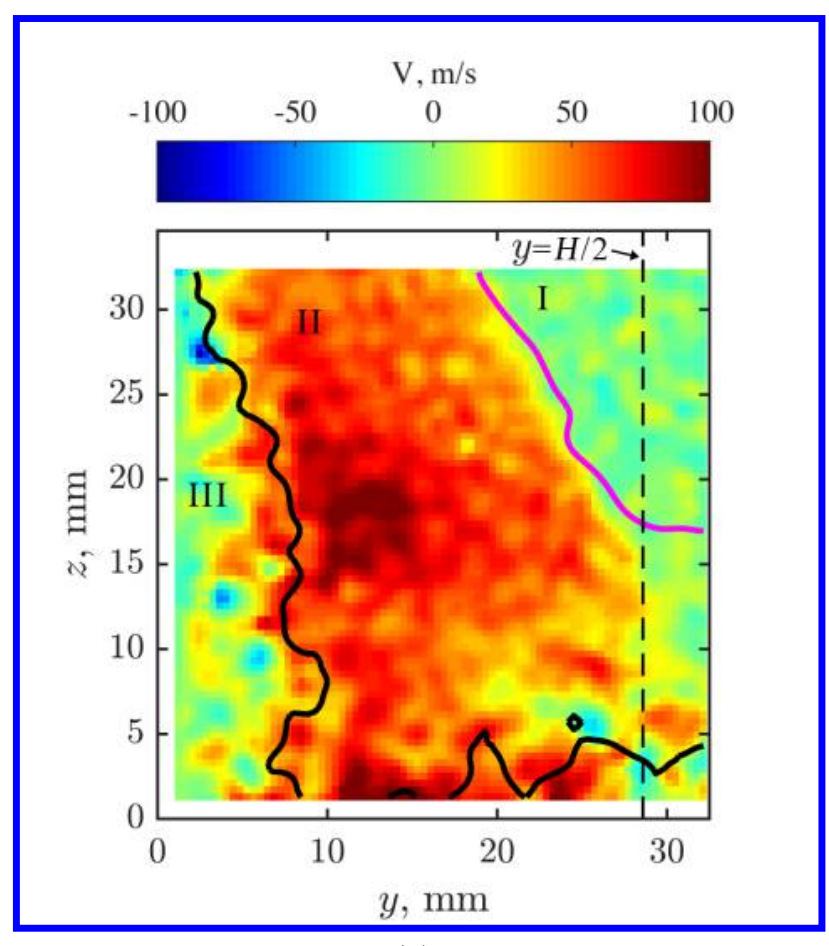

(b)

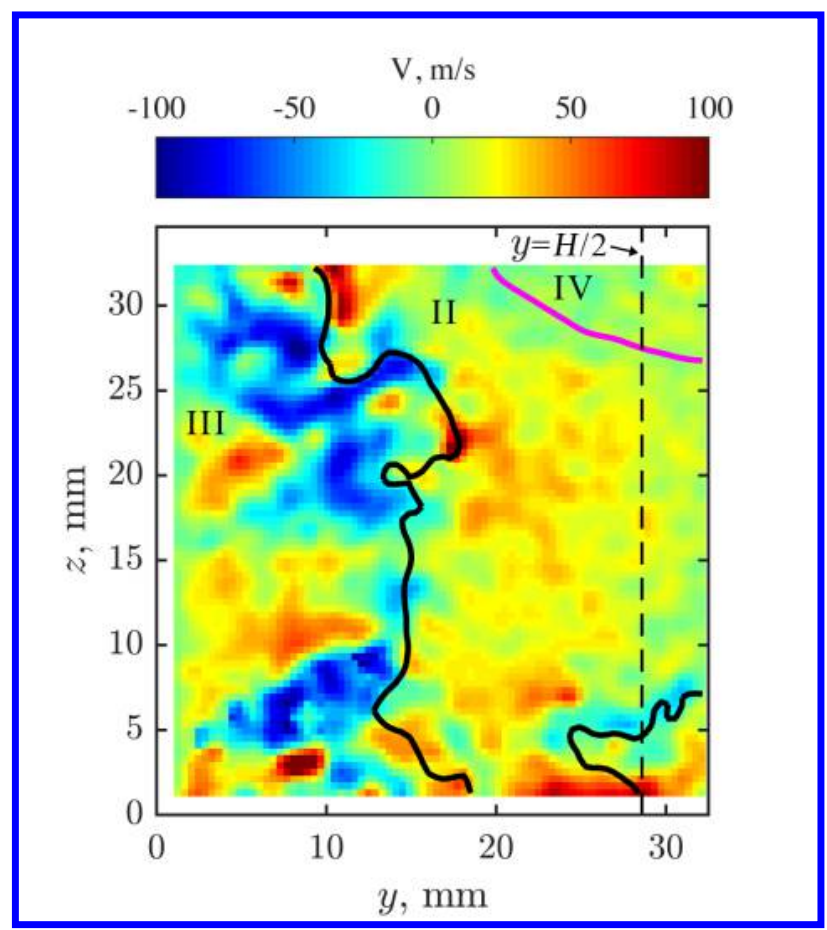

(d)

Figure 22. Instantaneous $V$ velocity field: (a) image plane $a$; (b) image plane $b$; (c) image plane $c$; (d) image plane $d$. 


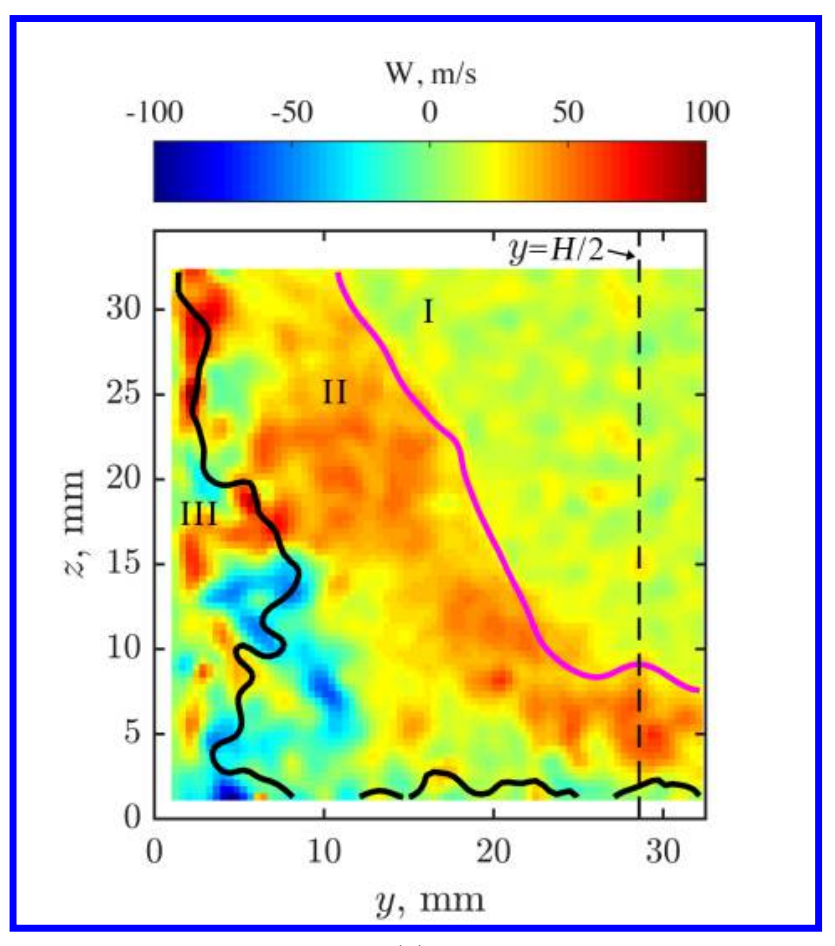

(a)

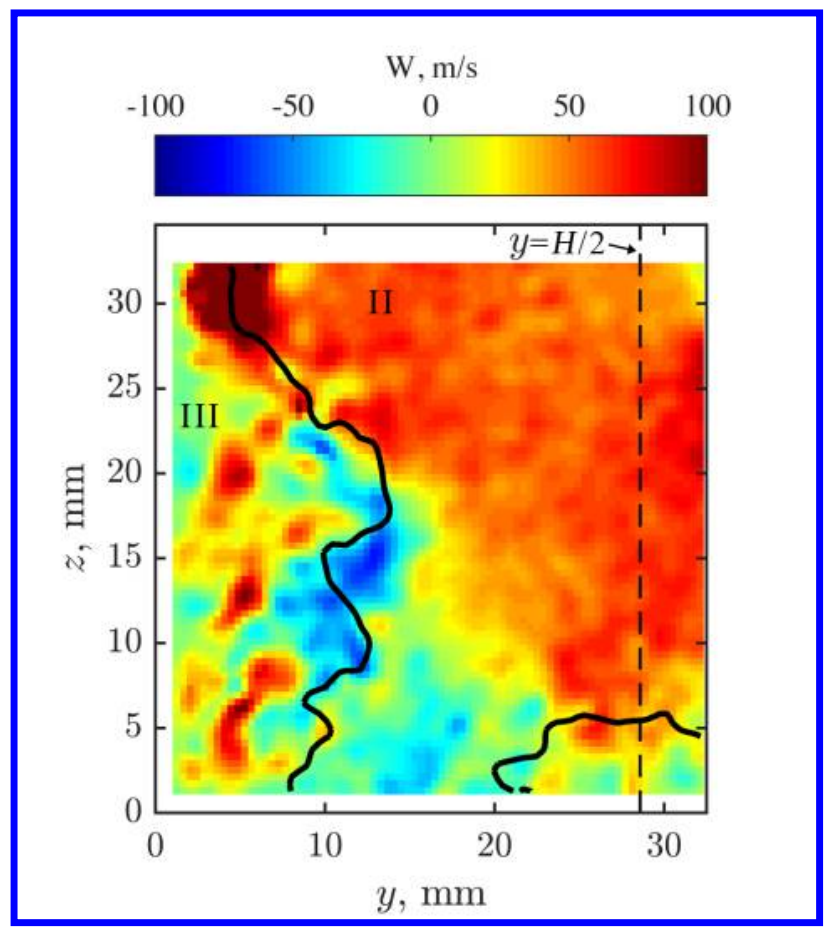

(c)

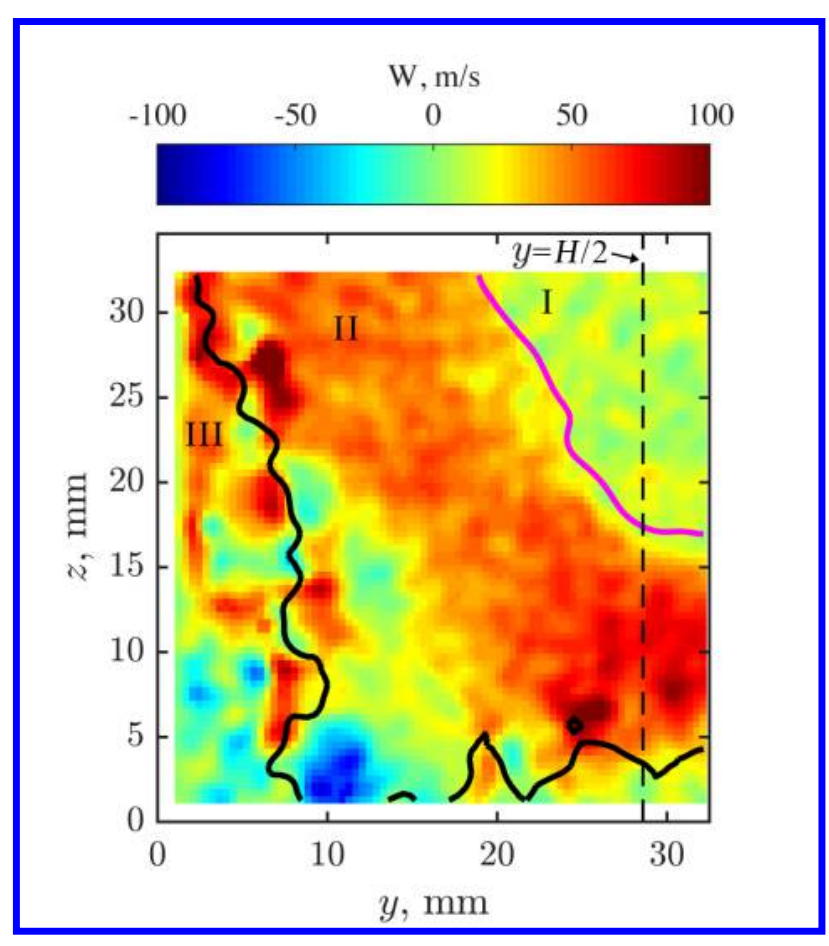

(b)

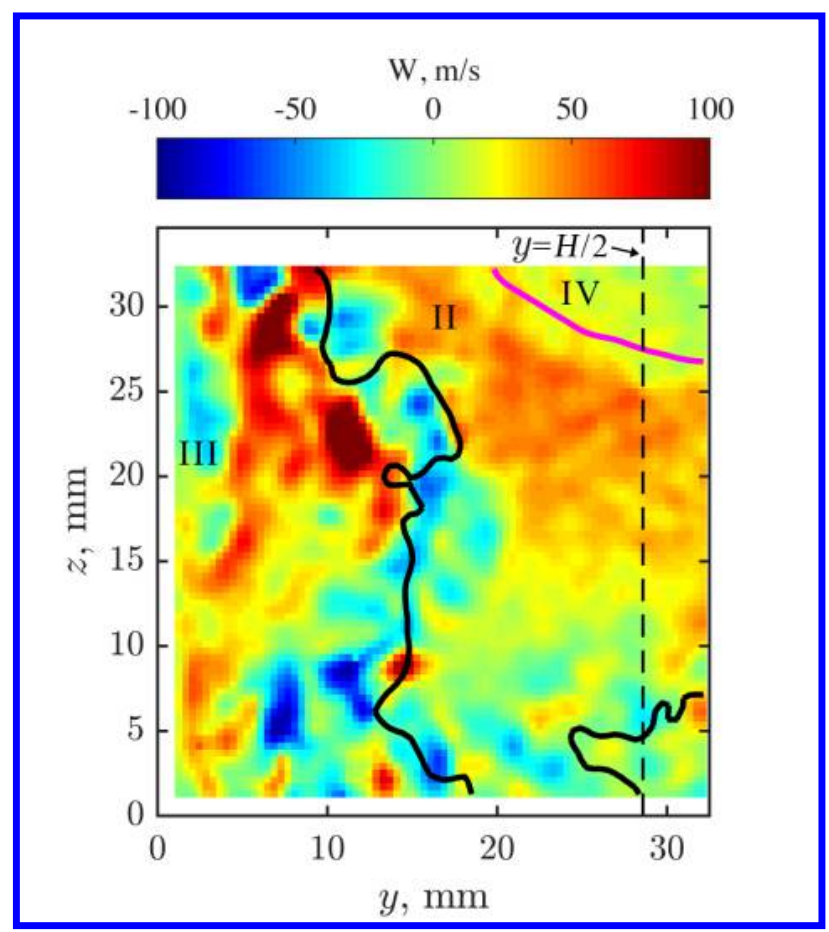

(d)

Figure 23. Instantaneous $W$ velocity field: (a) image plane $a$; (b) image plane $b$; (c) image plane $c$; (d) image plane $d$. 


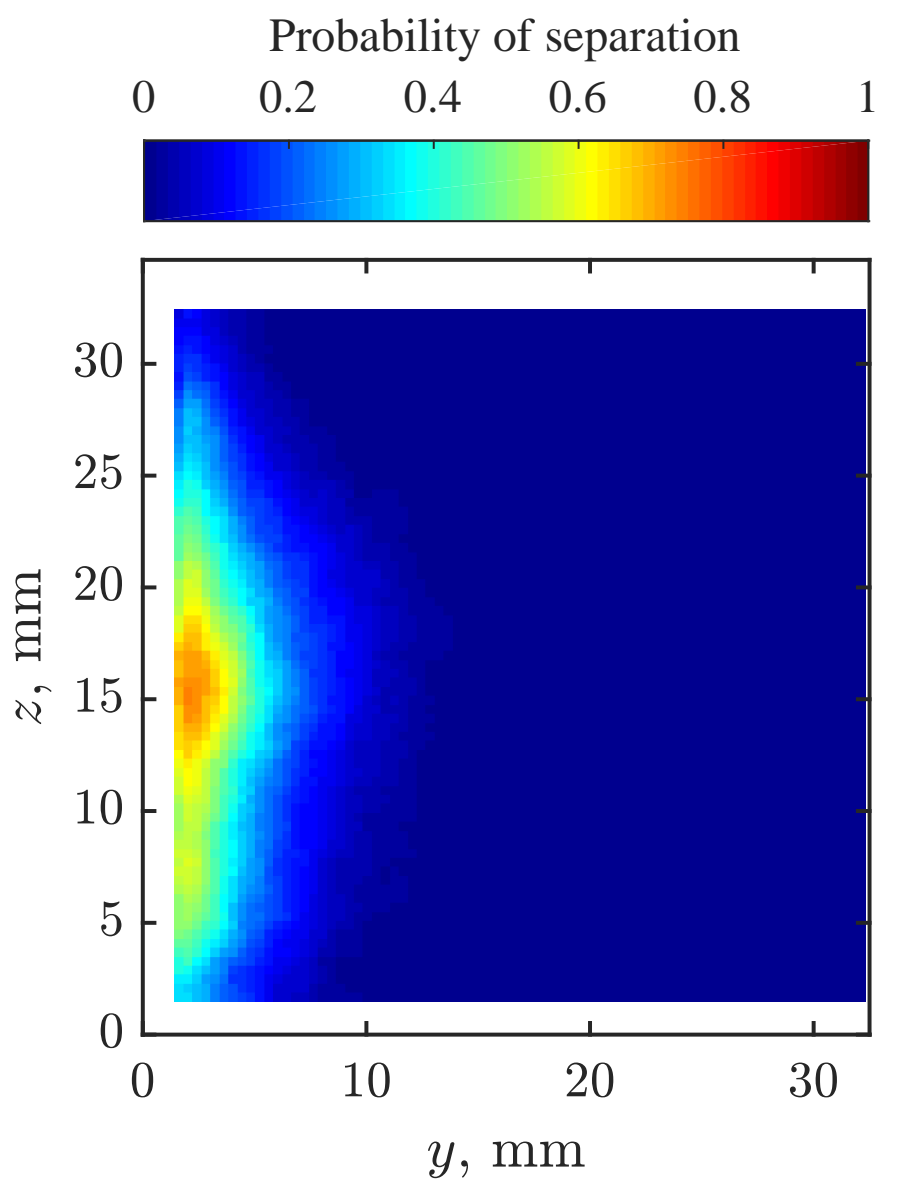

Figure 24. Probability of flow separation under the leading shock bottom-wall lambda foot. 


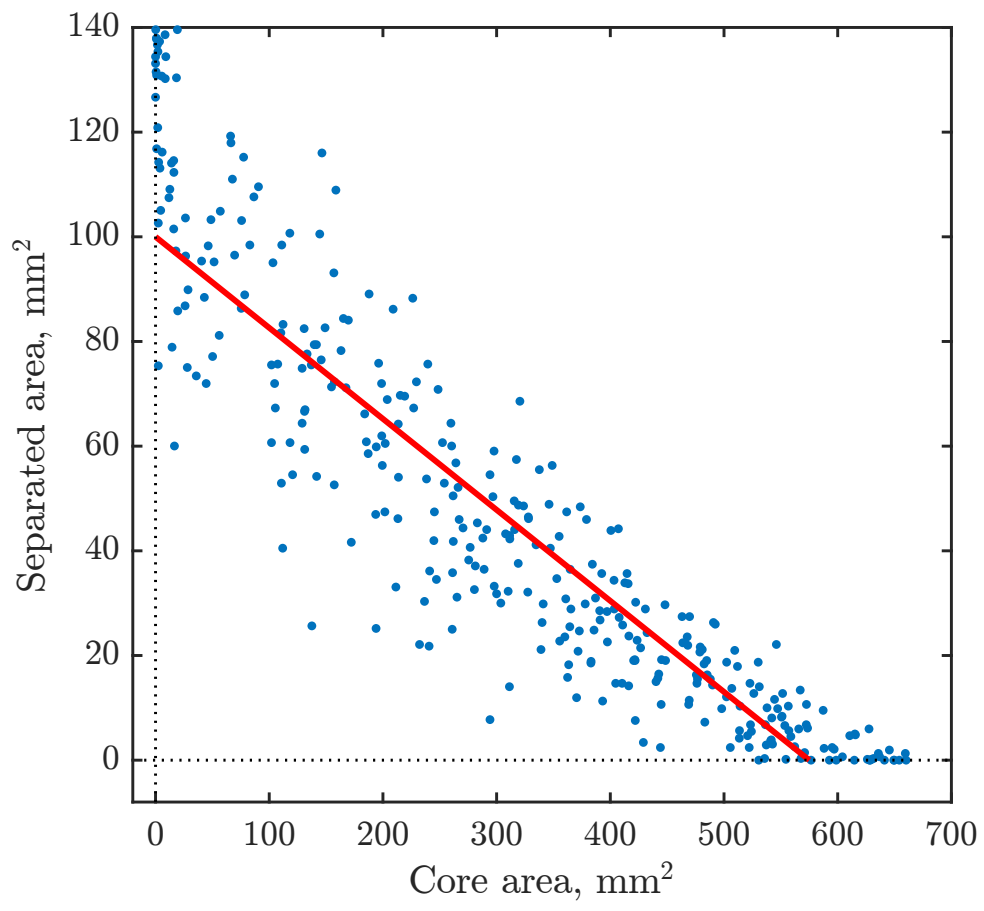

Figure 25. Separation area versus the core area of the flow. 


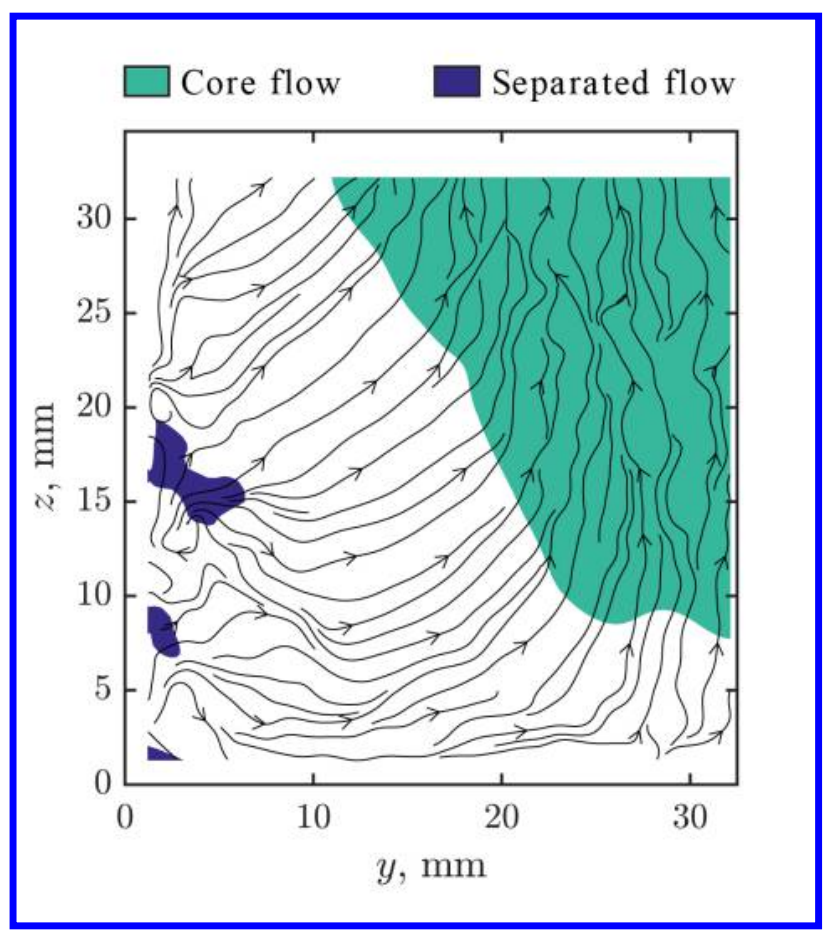

(a)

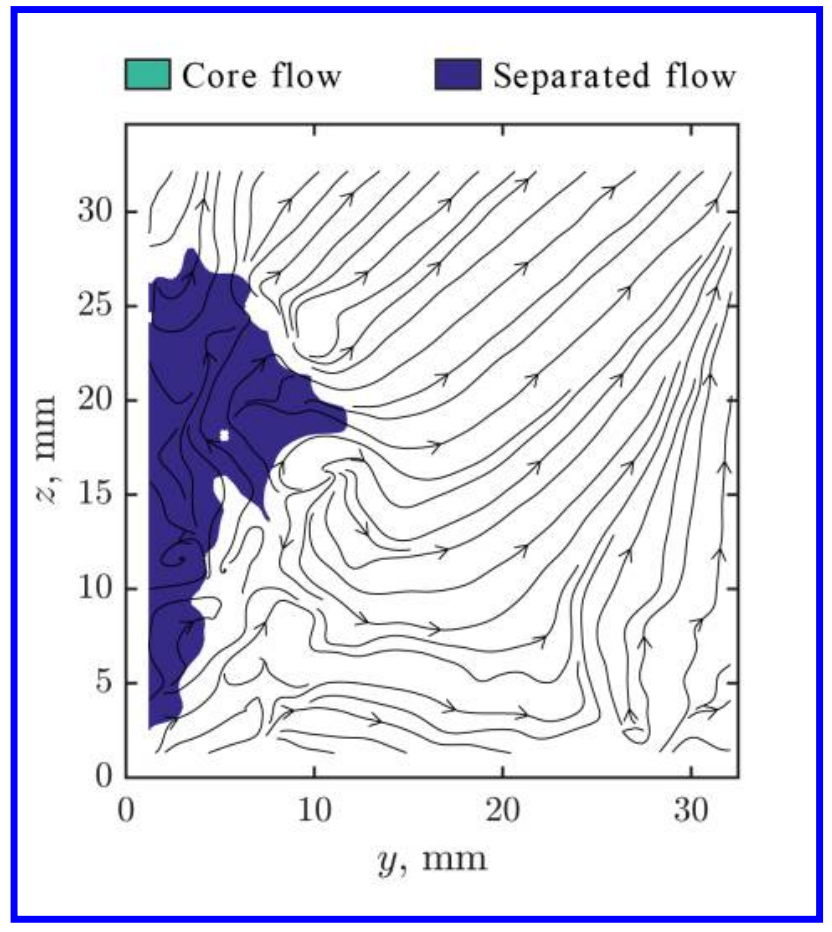

(c)

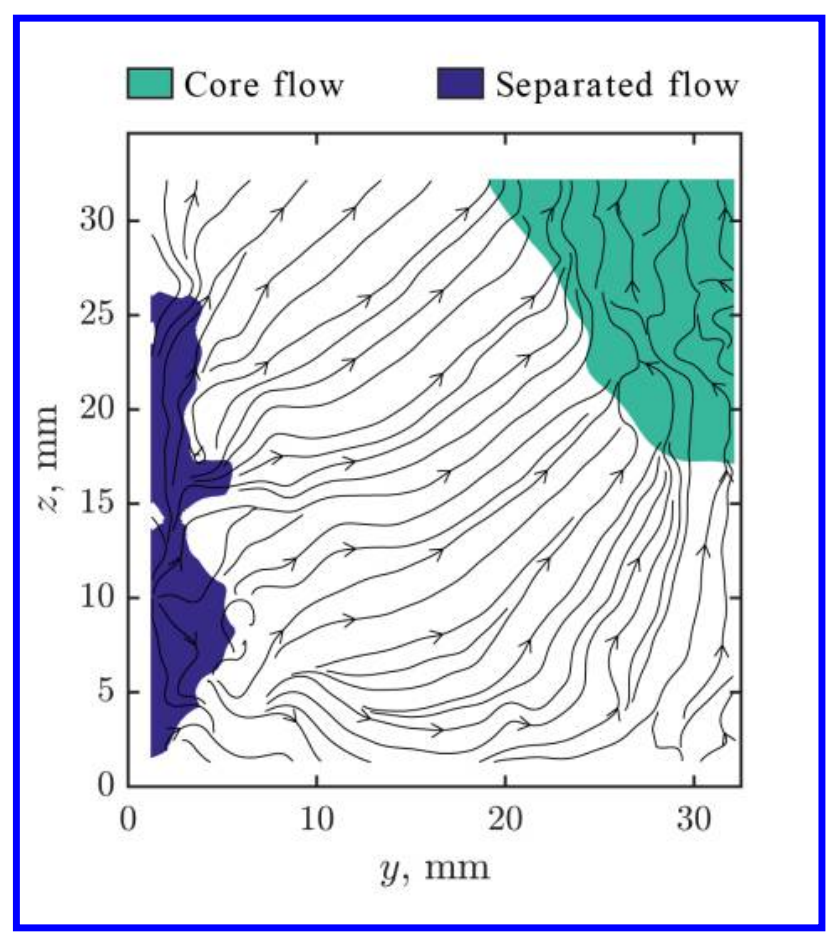

(b)

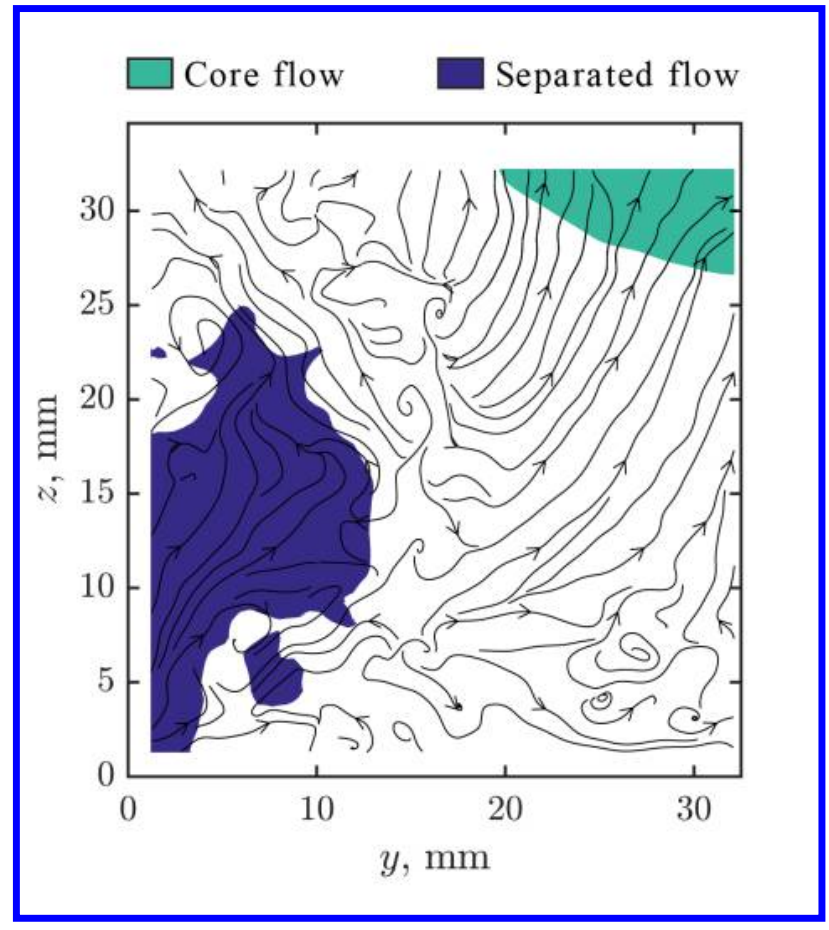

(d)

Figure 26. Streamlines: (a) image plane $a$; (b) image plane $b$; (c) image plane $c$; (d) image plane $d$. 


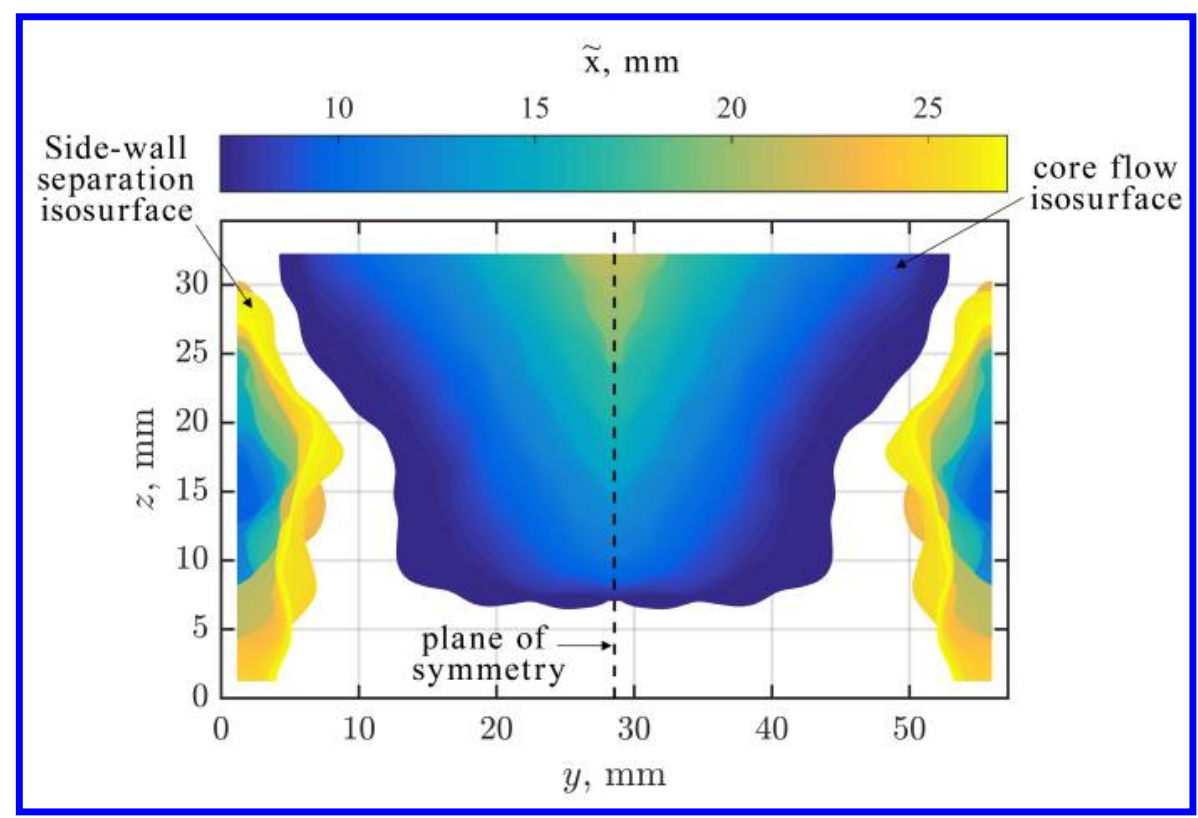

(a)

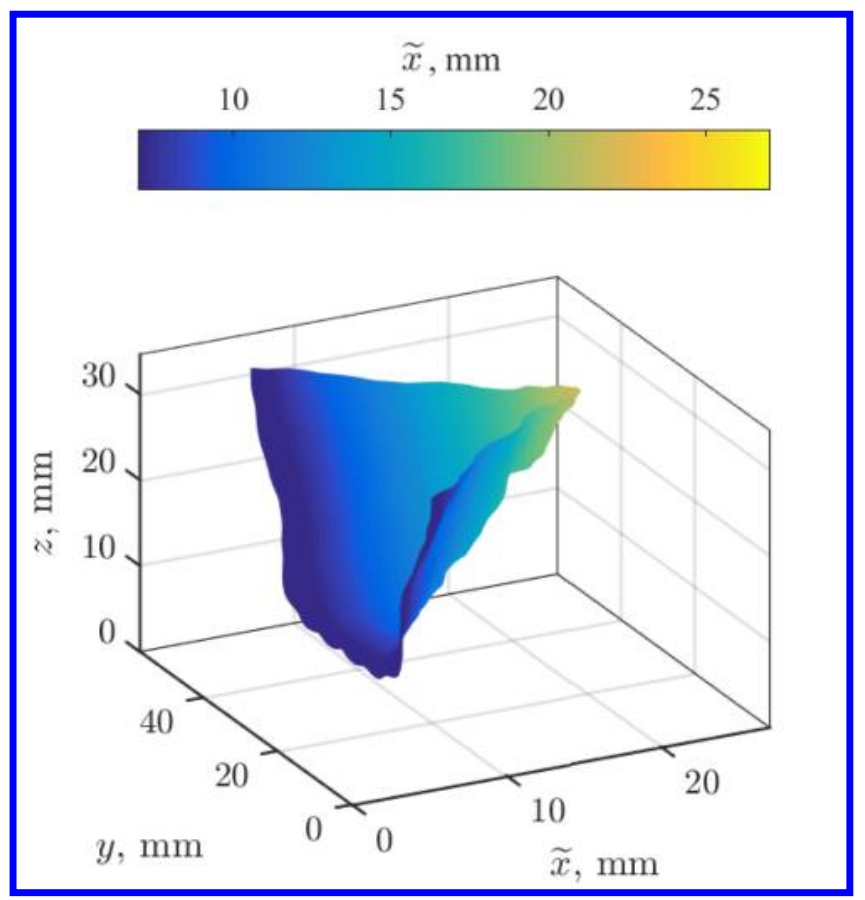

(b)

Figure 27. Shape of the core flow and separated region; (a) cross-sectional view; (b) perspective view. 


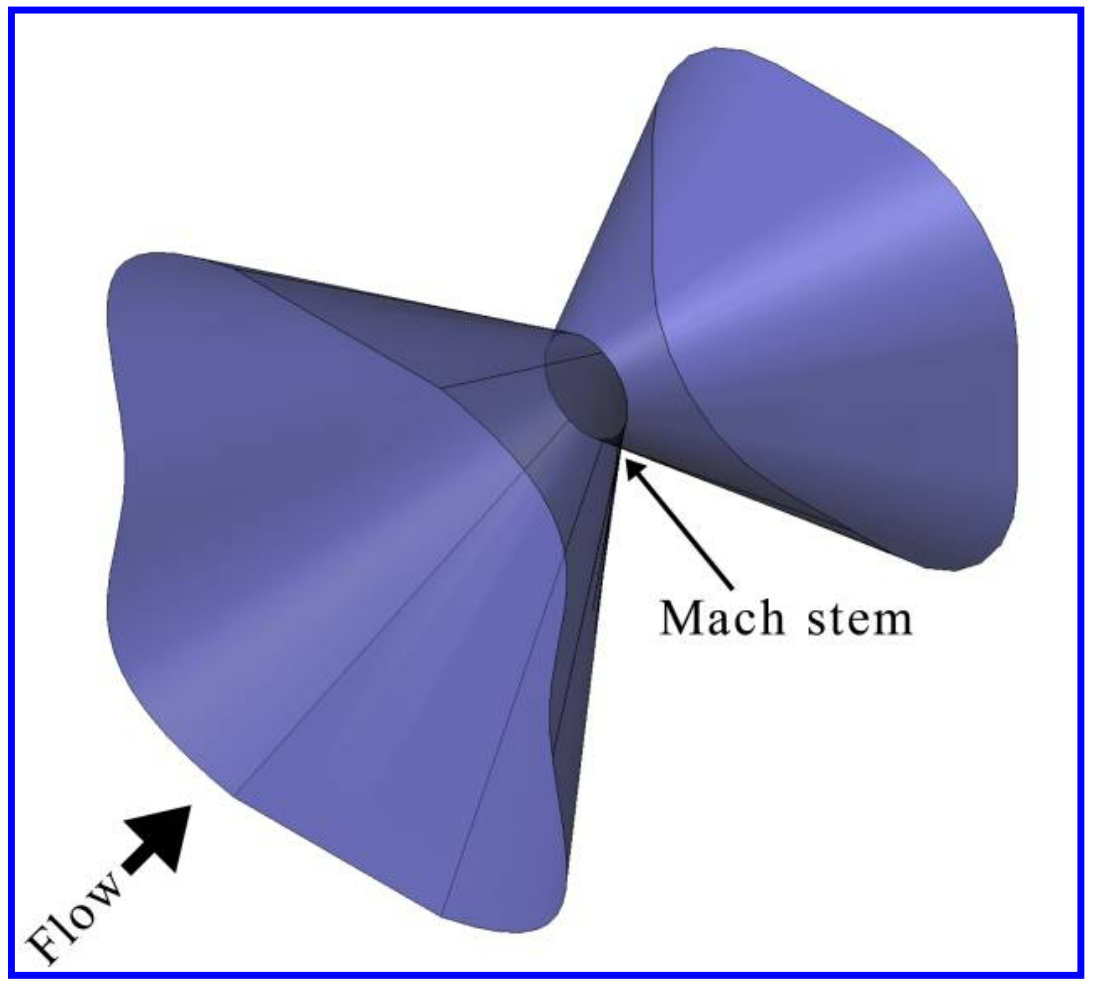

Figure 28. Three-dimensional model of the leading shock in the shock train. 
This article has been cited by:

1. Leon Vanstone, Joe Lingren, Noel T. Clemens. Supersonic Isolator Shock-Train Dynamics: Simple Physics-Based Model for Closed-Loop Control of Shock-Train Location . [Citation] [PDF] [PDF Plus]

2. Leon Vanstone, Kelley E. Hashemi, Joe Lingren, Maruthi R. Akella, Noel T. Clemens, Jeffrey Donbar, Sivaram Gogineni. ClosedLoop Control of Shock-Train Location in a Combusting Scramjet. Journal of Propulsion and Power, ahead of print1-8. [Abstract] [Full Text] [PDF] [PDF Plus]

3. Robin Hunt, James F. Driscoll, Mirko Gamba. Periodic forcing of a shock train in Mach 2.0 flow . [Citation] [PDF] [PDF Plus] 